To Appear in The Astrophysical Journal

Preprint typeset using LATEX style AASTeX6 v. 1.0

\title{
NEW NEUTRON-CAPTURE MEASUREMENTS IN 23 OPEN CLUSTERS. I. THE R-PROCESS
}

\author{
Jamie C. Overbeek \\ Indiana University Astronomy Department, Swain West 318, 727 East 3rd Street, Bloomington, IN 47405, USA; joverbee@indiana.edu
}

EILEEN D. FRIEL

Indiana University Astronomy Department, Swain West 318, 727 East 3rd Street, Bloomington, IN 47405, USA

HEATHER R. JACOBSON

Massachusetts Institute of Technology, Kavli Institute for Astrophysics and Space Research, Cambridge, MA 02139, USA

\begin{abstract}
Neutron-capture elements, those with $\mathrm{Z}>35$, are the least well-understood in terms of nucleosynthesis and formation environments. The rapid neutron-capture, or r-process, elements are formed in the environments and/or remnants of massive stars, while the slow neutron-capture, or s-process, elements are primarily formed in low-mass AGB stars. These elements can provide much information about Galactic star formation and enrichment, but observational data is limited. We have assembled a sample of 68 stars in 23 open clusters that we use to probe abundance trends for six neutron-capture elements (Eu, Gd, Dy, Mo, Pr, and Nd) with cluster age and location in the disk of the Galaxy. In order to keep our analysis as homogenous as possible, we use an automated synthesis fitting program, which also enables us to measure multiple (3-10) lines for each element. We find that the pure r-process elements (Eu, Gd, and Dy) have positive trends with increasing cluster age, while the mixed r- and s- process elements (Mo, Pr, and $\mathrm{Nd}$ ) have insignificant trends consistent with zero. $\mathrm{Pr}, \mathrm{Nd}, \mathrm{Eu}, \mathrm{Gd}$, and Dy have similar, slight (though mostly statistically significant) gradients of $\sim 0.04 \mathrm{dex} / \mathrm{kpc}$. The mixed elements also appear to have nonlinear relationships with $\mathrm{R}_{\mathrm{GC}}$.
\end{abstract}

Keywords: Galaxy: abundances, open clusters and associations, stars: abundances

\section{INTRODUCTION}

Various astronomical fields such as stellar evolution, the formation of the Galaxy, and galactic chemical evolution all require an understanding of stellar nucleosynthesis. Although the light elements, $\alpha$ - and Fe-peak element nucleosynthesis processes are relatively well understood, less theoretical and observational data are available for elements heavier than $\mathrm{Z} \sim 35$ which are collectively called the neutron-capture elements. These elements build in two main ways: via slow neutron capture (the s-process) and rapid neutron-capture (the rprocess), with most having contributions from both processes. Once an atomic nucleus captures a neutron, it will then undergo $\beta$-decay until it reaches the valley of $\beta$-stability. The slow neutron-capture process occurs in environments with relatively low neutron flux where $\beta$ decay occurs more quickly than successive neutron captures. The r-process occurs in high neutron flux environments where nuclei capture neutrons much more quickly than the timescale of $\beta$-decay. Thus, the two processes create different isotopes with varying efficiency.

The s-process is comprised of several components, the weak, the strong, and the main, with recent evidence for a light element primary process (Travaglio et al. 2004). s-process elements are produced in multiple astrophysical sites, although low-mass $\left(\mathrm{M}<4 \mathrm{M}_{\odot}\right)$ AGB stars are generally thought to be the most significant contributors. The site of r-process formation, however, has not yet been conclusively identified; core-collapse supernovae and neutron star-neutron star or neutron starblack hole mergers are the primary candidates (see, e.g., Burbidge et al. 1957; Freiburghaus et al. 1999; Vangioni et al. 2016). It has also been suggested that there may be two r-process sites, one for species below and one for species above $\mathrm{A} \sim 140$ (Wasserburg et al. 1996).

Open clusters (OCs) are useful tools for studying neutron-capture abundances in approximately solarmetallicity stars. Their ages and locations within the Galaxy are relatively easy to determine and cover a sig- 
nificant range in parameter space. Though much work has been done measuring r-process element abundances in metal-poor halo stars (e.g. Spite \& Spite 1978; Sneden et al. 1994; Honda et al. 2004; François et al. 2007), relatively few measurements of OC r-process abundances are present in the literature, mostly focusing on $\mathrm{Eu}$. Unfortunately, these Eu results are sometimes conflicting. Yong et al. (2012), using a sample of ten OCs plus four literature measurements, find a $[\mathrm{Eu} / \mathrm{Fe}]$ trend with age of $-0.01 \pm 0.01 \mathrm{dex} \mathrm{Gyr}^{-1}$, while Jacobson \& Friel (2013), using a sample of 19 OCs, find a trend in the opposite sense of +0.023 dex $\mathrm{Gyr}^{-1}$.

In order to better constrain the high-metallicity rprocess regime, we have assembled a sample of 68 stars in 23 OCs and measured abundances for six neutroncapture elements (three primarily r-process and three mixed $\mathrm{r}$ - and s-process elements) which we present here. S-process abundance measurements for this sample will be discussed in a forthcoming paper. The article is laid out as follows: in Section 2 we discuss our sample data, in Section 3 we describe atmospheric parameter determinations, in Section 4 we give the neutron-capture abundance measurements and dispersions, in Section 5 we discuss anomalous stars, Section 6 describes our error estimates, in Section 7 we draw comparisons to the literature and discuss trends and gradients, and in Section 8 we summarize our findings.

\section{OBSERVATIONS AND DATA}

\subsection{Previously Presented and Archival Data}

Our OC data set is comprised mainly of KPNO $4 \mathrm{~m}$, CTIO, and HET spectra taken in past observing runs (described in Friel et al. 2003, 2005; Jacobson et al. 2008, 2009; Friel et al. 2010; Jacobson \& Friel 2013). These are observations of red giants in Be $17, \mathrm{Be} 39, \mathrm{Cr}$ 261, M67, NGC 188, NGC 1193, NGC 1245, NGC 1817, NGC 1883, NGC 2141, NGC 2158, NGC 2194, NGC 2355, NGC 6939, and NGC 7142.

We have also obtained Keck spectra of five clusters, Be 18, Be 21, Be 22, Be 32, and PWM4, and CTIO spectra for Be 31, courtesy of D. Yong (see Yong et al. 2005, 2012, for details). We have McDonald 2.7m spectra of five stars in NGC 7789 courtesy of Michael Briley (private communication, 2013), and VLT UVES spectra of four stars in NGC 6192 from Laura Magrini (see Magrini et al. 2010, for details). We have recently taken additional spectra of two stars each in NGC 1817, NGC 2141, and NGC 6939 with the Apache Point Observatory (APO) $3.5 \mathrm{~m}$ (see below). We have 23 OCs total in our sample, which are summarized in Table 1 along with parameters, references, and sources of data. The signalto-noise of these data range from 60 to 180 per pixel, and the resolution ranges from $\mathrm{R} \sim 25,000$ (older KPNO data) to R 47,000 (UVES, Keck, and McDonald data). The Salaris et al. (2004) ages were used for all clusters where available (NGC 6192 was not included in their sample; we use an age of 0.18 Gyr from Clariá et al. 2006) except for Berkeley 31 and Collinder 261; these two clusters have seen significant variations in calculated ages. The first photometric study of Be 31 produced an age of $8 \mathrm{Gyr}$, which was steadily revised downwards over time to $\sim 2.5$ Gyr with a corresponding increase in $\mathrm{R}_{\mathrm{GC}}$ from $\sim 13$ to $16 \mathrm{kpc}$ (Guetter 1993; Janes \& Phelps 1994; Salaris et al. 2004; Hasegawa et al. 2004; Cignoni et al. 2011). We adopt the most recent age and galactocentric distance determinations, $2.6 \mathrm{Gyr}$, and $16.3 \mathrm{kpc}$, from Cignoni et al. (2011). Cr 261's calculated age has similarly decreased over time from $\sim 10$ to $6 \mathrm{Gyr}$, although its galactocentric radius has remained around $7.5 \mathrm{kpc}$ (Janes \& Phelps 1994; Carraro et al. 1998; Salaris et al. 2004; Bragaglia \& Tosi 2006). Again, we adopt the most recently determined parameters from Bragaglia \& Tosi (2006).

Be 31 also presents a challenge in membership determination. Friel et al. (2002) present low-resolution spectroscopic data for 17 stars in the field of Be 31, and find a mean cluster velocity of $+61 \pm 15 \mathrm{~km} \mathrm{~s}^{-1}$. Yong et al. (2005) find a similar cluster radial velocity of $+60 \pm$ $10 \mathrm{~km} \mathrm{~s}^{-1}$ for five stars in the field of Be 31, though they note the large scatter and that the CMD is complex and may have significant contamination from field stars. They analyze star 886 , with a radial velocity of $+56.6 \mathrm{~km} \mathrm{~s}^{-1}$, for abundances. Friel et al. (2010) observed two other stars in the field of Be 31 (260 and 1295) and found them to have velocities that differed by $19 \mathrm{~km} \mathrm{~s}^{-1}$, neither of which agreed with that of star 886 from Yong et al. (2005). In an effort to resolve the membership status of these stars, Friel (private communication, 2015) has observed a sample of 57 stars in the field of Be 31 with the Hydra multi-object spectrograph at WIYN ${ }^{1}$. These observations show a quite broad velocity distribution, but with a clear signature of the cluster velocity at $\sim 55 \mathrm{~km} \mathrm{~s}^{-1}$. This cluster velocity indicates that the two stars observed by Friel et al. (2010), are in fact, not members of the cluster, while the velocity of star 886 is coincident with the cluster mean velocity. We therefore restrict our analysis of Be 31 to star 886 .

\subsection{New APO Data}

We obtained additional APO spectra for several previously observed clusters to better define the dispersions in these clusters, which were found to be unusually large for

\footnotetext{
1 The WIYN Observatory is a joint facility of the University of Wisconsin-Madison, Indiana University, the National Optical Astronomy Observatory and the University of Missouri.
} 
Table 1. Clusters in the Sample

\begin{tabular}{|c|c|c|c|c|c|c|c|c|}
\hline Cluster & $\begin{array}{c}1 \\
\text { (deg.) }\end{array}$ & $\begin{array}{c}\text { b } \\
\text { (deg.) }\end{array}$ & $\begin{array}{c}\mathrm{d} \odot \\
(\mathrm{kpc})\end{array}$ & $\begin{array}{c}\text { Age } \\
\text { (Gyr) }\end{array}$ & $\begin{array}{c}\mathrm{R}_{\mathrm{GC}}{ }^{\mathrm{a}} \\
(\mathrm{kpc})\end{array}$ & Ref. $^{\text {b }}$ & $\begin{array}{l}\text { No. of } \\
\text { Stars }\end{array}$ & Telescope \\
\hline Be 17 & 175.7 & -3.7 & 2.7 & 10.1 & 11.2 & F05 & 3 & KPNO $4 \mathrm{~m}$ \\
\hline Be 18 & 163.6 & 5.0 & 5.4 & 5.7 & 13.7 & $\mathrm{Y} 12$ & 2 & Keck \\
\hline Be 21 & 186.8 & -2.5 & 6.2 & 2.2 & 14.7 & $\mathrm{Y} 12$ & 2 & Keck \\
\hline Be 22 & 199.9 & -8.1 & 6.2 & 4.3 & 14.4 & $\mathrm{Y} 12$ & 2 & Keck \\
\hline $\operatorname{Be} 31^{\mathrm{c}}$ & 206.2 & 5.1 & 8.3 & 2.6 & 16.3 & $\mathrm{C} 11$ & 1 & CTIO $4 \mathrm{~m}$ \\
\hline Be 32 & 208.0 & 4.4 & 3.4 & 5.9 & 11.6 & FJP10 & 2,2 & KPNO 4m, Keck \\
\hline Be 39 & 223.5 & 10.1 & 4.3 & 7.0 & 11.9 & FJP10 & 4 & KPNO $4 \mathrm{~m}$ \\
\hline Cr $261^{\mathrm{c}}$ & 301.7 & -5.6 & 2.7 & 6.0 & 7.4 & ВТ06 & 2 & CTIO $4 \mathrm{~m}$ \\
\hline M67 & 215.6 & 31.7 & 0.9 & 4.3 & 9.1 & FJP10 & 3 & $\mathrm{KPNO} 4 \mathrm{~m}$ \\
\hline NGC 188 & 122.8 & 22.5 & 1.7 & 6.3 & 9.4 & FJP10 & 4 & $\mathrm{KPNO} 4 \mathrm{~m}$ \\
\hline NGC 1193 & 146.8 & -12.2 & 5.8 & 4.2 & 13.6 & FJP10 & 1 & HET \\
\hline NGC 1245 & 146.6 & -8.9 & 3.0 & 1.1 & 11.1 & J11 & 4 & KPNO $4 \mathrm{~m}$ \\
\hline NGC 1817 & 186.1 & -13.1 & 1.5 & 1.1 & 10.0 & J09 & 2,2 & KPNO $4 \mathrm{~m}$, APO $3.5 \mathrm{~m}$ \\
\hline NGC 1883 & 163.1 & 6.2 & 3.9 & 0.7 & 12.3 & J09 & 2 & KPNO $4 \mathrm{~m}$ \\
\hline NGC 2141 & 198.0 & -5.8 & 3.9 & 2.4 & 12.2 & J09 & 2,2 & $\mathrm{KPNO} 4 \mathrm{~m}$, APO $3.5 \mathrm{~m}$ \\
\hline NGC 2158 & 186.6 & 1.8 & 4.0 & 1.9 & 12.5 & J09 & 1 & KPNO $4 \mathrm{~m}$ \\
\hline NGC 2194 & 197.3 & -2.3 & 1.9 & 0.9 & 10.3 & $\mathrm{~J} 11$ & 2 & KPNO $4 \mathrm{~m}$ \\
\hline NGC 2355 & 203.4 & 11.8 & 1.9 & 0.8 & 10.2 & $\mathrm{~J} 11$ & 3 & KPNO $4 \mathrm{~m}$ \\
\hline NGC $6192^{\mathrm{c}}$ & 340.7 & 2.1 & 1.5 & 0.2 & 7.1 & $\mathrm{C} 06$ & 4 & VLT \\
\hline NGC 6939 & 95.9 & 12.3 & 1.8 & 1.2 & 8.9 & A04 & 4,2 & KPNO $4 \mathrm{~m}$, APO $3.5 \mathrm{~m}$ \\
\hline NGC 7142 & 105.0 & 9.0 & 1.9 & 4.0 & 9.2 & J08 & 4 & KPNO $4 \mathrm{~m}$ \\
\hline NGC 7789 & 115.5 & -5.4 & 2.2 & 1.8 & 9.6 & $\mathrm{~J} 11$ & 5 & McDonald $2.7 \mathrm{~m}$ \\
\hline PWM 4 & 116.0 & 0.3 & 7.2 & 7.0 & 13.3 & Y12 & 1 & Keck \\
\hline \multicolumn{9}{|c|}{${ }^{a} \mathrm{R}_{\mathrm{GC}, \odot}=8.5 \mathrm{kpc}$} \\
\hline \multicolumn{9}{|c|}{$\begin{array}{l}{ }^{b} \text { References for distances: C11 = Cignoni et al. (2011); BT06 = Bragaglia \& Tosi (2006); C06 = Clariá } \\
\text { et al. (2006); F05 = Friel et al. (2005); FJP10 = Friel et al. (2010); J08 = Jacobson et al. (2008); } \\
\text { J09 = Jacobson et al. (2009); J11 = Jacobson et al. (2011); Y05 = Yong et al. (2005); Y12 = Yong } \\
\text { et al. (2012) }\end{array}$} \\
\hline
\end{tabular}

some elements in Jacobson \& Friel (2013). Our target selection for the new APO observations of NGC 1817 and NGC 6939 was based on WIYN 3.5m observations of the two clusters presented in Jacobson et al. (2011) and Jacobson et al. (2007), respectively, which confirmed these stars as members based on radial velocity. Our two targets in NGC 2141 were selected based on radial velocity confirmation from Yong et al. (2005). Our APO data were taken on the nights of 16 Nov. 2014 (NGC 1817 and NGC 6939), 6, 15, 26, and 28 of Jan. 2015 (NGC 2141). We used the ARC Echelle Spectrograph on standard settings (2048x2048 SITe CCD, 3200-10000, R $32000)$ to obtain spectra of two stars per cluster. Because we targeted red giant stars, our signal-to-noise was unfortunately limited in the blue, but we were able to measure lines to $\sim 4500 \AA$ for each spectrum. Data were reduced using the ARCES reduction guide by Julie
Thorburn ${ }^{2}$. The signal-to-noise of our combined and reduced spectra at $6000 \AA$ ranges from 75 to 90 . Details for these observations are summarized in Table 2.

Radial velocities for our APO spectra were measured with FXCOR in $\mathrm{IRAF}^{3}$ by measuring a radial velocity for each aperture, then taking the weighted mean of the aperture velocities and errors. However, the error on the weighted mean of these values significantly underestimates the errors caused by night-to-night zero point variations. For the star we observed over three nights, N2141 514, we find that measured heliocentric radial velocities for different nights have a dispersion of 0.6

\footnotetext{
2 http://www.apo.nmsu.edu/arc35m/Instruments/ ARCES/images/echelle_data_reduction_guide.pdf

${ }^{3}$ IRAF is distributed by the National Optical Astronomy Observatory, which is operated by the Association of Universities for Research in Astronomy, Inc., under cooperative agreement with the National Science Foundation.
} 
$\mathrm{km} \mathrm{s}^{-1}$, so we adopt this as the error on our velocities. We generally find good agreement within the errors for available literature radial velocities for N1817 and N2141 stars; these have differences less than $2 \mathrm{~km} \mathrm{~s}^{-1}$ with Mermilliod et al. (2003) and Jacobson et al. (2011) for N1817 and Yong et al. (2005) for N2141. However, our N6939 velocities are lower than those found in Milone (1994) and Jacobson et al. (2007) by several $\mathrm{km} \mathrm{s}^{-1}$. It is possible that both stars may be binaries, or this may be due to differences in zero-point corrections, but our measured radial velocities are close enough to those in the literature that we are confident these stars are cluster members. It is also worth noting that Mermilliod et al. (2003) classify N1817 1456 as a possible spectroscopic binary due to the significant dispersion in CORAVEL radial velocity measurements, but we do not see any binarity in the line profiles of our spectrum.

\section{ATMOSPHERIC PARAMETERS AND IRON}

Atmospheric parameters and Fe abundances for all stars described in Jacobson \& Friel (2013) are taken from that work so we will not describe them here. In order to remain consistent with our internal abundance scale, we have calculated our own atmospheric parameters and iron abundances for all stars we have not previously analyzed. We used spherical MARCS ${ }^{4}$ model atmospheres (Gustafsson et al. 2008) with the 2010 version of MOOG (Sneden 1973) for all abundance calculations. We selected a set of relatively unblended iron lines from the Gaia-ESO Survey (GES) to measure equivalent widths (Heiter et al. 2015); Fe lines, atomic data, and equivalent widths are listed in Table 3.

Echelle $[\mathrm{Fe} / \mathrm{H}]$ abundances taken from Jacobson \& Friel (2013) were calculated using a linelist with Arcturus-based $\log (\mathrm{gf})$ values, different than the GES linelist we present here for the newest stellar abundance measurements. In a previous abundance study of NGC 7789 using lower-resolution WIYN Hydra spectra, we demonstrated that the differences between the two linelists are small and do not give rise to any systematic differences in atmospheric parameters (Overbeek et al. 2015). We did find that for the NGC 7789 data, using the Arcturus-based linelist resulted in a $\sim 0.1$ dex higher $[\mathrm{Fe} / \mathrm{H}]$, but comparing the three clusters for which we have iron abundances based on both linelists (NGC 1817, NGC 2141, and NGC 6939) we find no difference in the cluster $[\mathrm{Fe} / \mathrm{H}]$ determined with the Arcturus-based vs. the GES $\log (\mathrm{gf})$ values.

Starting from the literature atmospheric parameters for Be 31 and NGC 6192, or typical previously determined clump parameters for NGC 7789, NGC 1817,

${ }^{4}$ Retrieved from http://marcs.astro.uu.se/
NGC 2141, and NGC 6939, we determined parameters spectroscopically with the typical method: adjusting temperature to minimize abundance trends with excitation potential, adjusting microturbulent velocity to minimize abundance trends with reduced equivalent width, adjusting the gravity to match abundances from Fe I and Fe II, and repeating until a good solution is found. We have also re-calculated spectroscopic parameters for the two warmer Cr 261 stars from Friel et al. (2003) since the Fe linelist used here is significantly different; setting the continuum was difficult for the cooler Cr 261 stars and resulted in large abundance errors, so we do not include them in this sample. Derived atmospheric parameters and $\mathrm{Fe}$ abundances can be found in Table 4 , along with literature parameters (where available) for comparison.

Our atmospheric parameters for Be 31 match those in Yong et al. (2005) well; the Fe abundance differs by 0.15 dex, within the errors for the two measurements. Parameters for Collinder 261 also match those in the literature, and Fe differences are again within the expected error. Our effective temperatures for NGC 6192 are systematically $100 \mathrm{~K}$ below Magrini et al. (2010), and our $\log (\mathrm{g})$ values are $\sim 0.3$ dex lower, although Fe abundances align well. Parameters for two overlapping stars between our sample and Tautvaišiené et al. (2005) also have very similar parameters and abundances. Our microturbulent velocities are systematically low compared to all literature values by about $0.2 \mathrm{~km} \mathrm{~s}^{-1}$.

Our derived $\mathrm{Fe}$ abundance for Be 31 compares well with literature values; the cluster has been consistently estimated to have a subsolar $[\mathrm{Fe} / \mathrm{H}] \sim-0.4$ dex as estimated by photometry (Guetter 1993; Hasegawa et al. 2004) and low-resolution spectroscopy (Friel et al. 2002). For Cr 261, Friel et al. (2003) find slightly subsolar Fe abundances, but Carretta et al. (2005), De Silva et al. (2007), and Mishenina et al. (2015) all find solar Fe abundances. Many previous spectroscopy and photometry studies have placed NGC 7789 at solar or slightly sub-solar metallicities (e.g., Pilachowski 1985; Twarog et al. 1997; Schönberner et al. 2001; Friel et al. 2002; Tautvaišienè et al. 2005; Pancino et al. 2010; Overbeek et al. 2015). Though our sample size per cluster is smaller than many of these studies, we can place some confidence in our derived $\mathrm{Fe}$ abundances.

We determine atmospheric parameter errors by taking into account both internal uncertainties (due to residual slopes with excitation potential and reduced equivalent widths for temperatures and microturbulent velocities and differences between FeI and FeII abundances for gravities) and systematic differences between our parameters and literature values. The residual slopes in abundance with excitation potential are small, on the order of $0.005 \mathrm{dex} \mathrm{eV}^{-1}$, and the typical uncertainty 
Table 2. APO Spectroscopic Observations

\begin{tabular}{|c|c|c|c|c|c|c|c|c|c|c|c|}
\hline Cluster & Star & $\begin{array}{l}\alpha_{J 2000} \\
(\mathrm{~h}: \mathrm{m}: \mathrm{s})\end{array}$ & $\begin{array}{l}\delta_{J 2000} \\
(\mathrm{~d}: \mathrm{m}: \mathrm{s})\end{array}$ & V & $\begin{array}{l}\text { Phot. } \\
\text { Ref. }^{\text {a }}\end{array}$ & $\begin{array}{c}\text { Exp. UT } \\
\text { Date }\end{array}$ & $\begin{array}{l}\text { Exp. } \\
\text { Time }\end{array}$ & $\begin{array}{c}\text { Lit. } \mathrm{V}_{r} \\
\left(\mathrm{~km} \mathrm{~s}^{-1}\right)\end{array}$ & $\begin{array}{c}\mathrm{V}_{r} \\
\text { Ref. }^{a}\end{array}$ & $\begin{array}{c}\mathrm{V}_{r} \\
\left(\mathrm{~km} \mathrm{~s}^{-1}\right)\end{array}$ & $\mathrm{S} / \mathrm{N}$ \\
\hline N1817 & 206 & 05:13:01.80 & $16: 41: 14.4$ & 11.92 & M03 & 20141116 & $5 \times 600 \mathrm{~s}$ & $65.5 \pm 0.4$ & M03 & 64.4 & 79 \\
\hline N1817 & 1456 & $05: 12: 32.83$ & $16: 28: 25.3$ & 11.43 & M03 & 20141116 & $5 \times 600 \mathrm{~s}$ & $66.0 \pm 1.0$ & M03 & 64.7 & 79 \\
\hline \multirow[t]{3}{*}{ N2141 } & 514 & 06:03:00.00 & $10: 32: 24.0$ & 14.09 & Y05 & 20150107 & $3 \times 3000 s$ & $23.3 \pm 0.9$ & Y05 & 23.2 & 76 \\
\hline & & & & & & 20150116 & $3 \times 3000 s$ & & & 24.1 & \\
\hline & & & & & & 20150127 & $1 \times 3000 \mathrm{~s}$ & & & 24.3 & \\
\hline \multirow[t]{2}{*}{ N2141 } & 1821 & 06:03:07.60 & $10: 26: 48.0$ & 14.13 & Y05 & 20150127 & $2 \times 3000 \mathrm{~s}$ & $24.8 \pm 1.0$ & Y05 & 25.3 & 76 \\
\hline & & & & & & 20150129 & $5 \times 3000 \mathrm{~s}$ & & & 25.4 & \\
\hline N6939 & 53 & 20:31:01.90 & $60: 38: 11.6$ & 12.78 & MN07 & 20141116 & $4 \times 1800 \mathrm{~s}$ & $-20.1 \pm 0.7$ & M94 & -23.6 & 93 \\
\hline N6939 & 65 & 20:31:05.98 & $60: 42: 13.8$ & 12.68 & MN07 & 20141116 & $4 \times 1800 \mathrm{~s}$ & $-18.6 \pm 0.6$ & M94 & -20.7 & 90 \\
\hline
\end{tabular}

${ }^{a}$ References for literature photometry and/or radial velocities: M03 = Mermilliod et al. (2003); MN07 = Maciejewski \& Niedzielski (2007); M94 = Milone (1994); Y05 = Yong et al. (2005)

Table 3. Fe Linelist Parameters and Equivalent Width Measurements ${ }^{\mathrm{a}}$

\begin{tabular}{|c|c|c|c|c|c|c|c|c|c|c|}
\hline $\begin{array}{c}\lambda \\
\operatorname{colhead}(\AA)\end{array}$ & Sp. & $\begin{array}{l}\text { E.P. } \\
(\mathrm{eV})\end{array}$ & $\log (\mathrm{gf})$ & $\begin{array}{c}\text { Be31 } 886 \\
\text { EQW (mÅ) }\end{array}$ & $\begin{array}{l}\text { Cr261 } 1045 \\
\text { EQW }(m \AA)\end{array}$ & $\begin{array}{l}\text { Cr261 } 1080 \\
\text { EQW }(m \AA)\end{array}$ & $\begin{array}{c}\text { N1817 } 206 \\
\text { EQW }(m \AA)\end{array}$ & $\begin{array}{l}\text { N1817 } 1456 \\
\text { EQW }(m \AA)\end{array}$ & $\begin{array}{c}\mathrm{N} 2141514 \\
\mathrm{EQW}(\mathrm{m} \AA)\end{array}$ & $\begin{array}{l}\mathrm{N} 21411821 \\
\mathrm{EQW}\end{array}$ \\
\hline 5242.49 & 26.0 & 3.63 & -0.967 & $\ldots$ & $\ldots$ & $\ldots$ & 122.6 & 128.1 & 130.9 & 117.8 \\
\hline 5365.40 & 26.0 & 3.57 & -1.020 & $\ldots$ & $\ldots$ & 111.3 & 127.4 & $\ldots$ & $\ldots$ & $\ldots$ \\
\hline 5379.57 & 26.0 & 3.69 & -1.514 & $\ldots$ & 92.5 & 93.9 & 106.5 & 104.8 & 110.7 & 98.3 \\
\hline 5417.03 & 26.0 & 4.42 & -1.580 & $\ldots$ & 67.8 & 57.2 & 57.3 & 68.6 & 62.2 & 51.3 \\
\hline 5466.99 & 26.0 & 3.57 & -2.233 & $\ldots$ & 79.3 & 73.3 & 77.0 & 88.8 & 85.1 & 76.9 \\
\hline 5633.95 & 26.0 & 4.99 & -0.230 & 76.0 & 89.6 & 84.9 & 90.1 & 88.4 & 92.9 & 84.0 \\
\hline 5662.52 & 26.0 & 4.18 & -0.447 & 102.6 & 118.2 & 114.8 & 123.5 & 129.5 & $\ldots$ & 115.9 \\
\hline 5701.54 & 26.0 & 2.56 & -2.193 & 117.5 & 143.7 & 138.3 & 132.7 & 139.0 & 158.3 & 135.0 \\
\hline 5705.46 & 26.0 & 4.30 & -1.355 & 70.3 & 73.8 & 77.2 & 70.9 & 79.6 & 82.9 & 75.4 \\
\hline 5753.12 & 26.0 & 4.26 & -0.623 & 86.0 & 104.9 & 102.5 & 102.8 & 117.1 & 111.9 & 108.0 \\
\hline
\end{tabular}

$a_{\text {Table }} 3$ is published in its entirety in the electronic edition of the AJ. A portion is shown here for guidance regarding its form and content.

on the slopes is $\sim 0.015$ dex $\mathrm{eV}^{-1}$, which correspond to changes in temperatures of $\pm 20 \mathrm{~K}$ and $\pm 75 \mathrm{~K}$ for a star with an effective temperature of $4500 \mathrm{~K}$. However, the typical difference between temperatures from this work and literature values in Table 4 is about $100 \mathrm{~K}$, so we estimate our temperature errors conservatively as $\pm 100 \mathrm{~K}$. For microturbulent velocity, the typical slope and error on the slope of abundance with reduced equivalent width are 0.02 and 0.09 , which correspond to differences in v.t. of 0.02 and $0.08 \mathrm{~km} \mathrm{~s}^{-1}$, but the median difference between our values and literature values is $0.2 \mathrm{~km} \mathrm{~s}^{-1}$, so we adopt $0.2 \mathrm{~km} \mathrm{~s}^{-1}$ for the error on v.t. Differences between our Fe I and Fe II values are no more than \pm 0.02 dex, corresponding to an internal uncertainty in $\log (\mathrm{g})$ of 0.1 dex, but again the differences between studies are larger, so we adopt the median difference between our and literature $\log (\mathrm{g})$ of 0.2 dex as the error on the gravities. Our $[\mathrm{Fe} \mathrm{I} / \mathrm{H}]$ dispersions are typically $0.10-0.15$ dex, and Fe II dispersions are similar. We discuss the impact of these errors on neutron-capture abundances in Section 6.

\section{NEUTRON-CAPTURE ABUNDANCE MEASUREMENTS}

We used the MOOG LTE spectral synthesis program to measure all neutron-capture elements presented here (Sneden 1973). We have used previously determined $\mathrm{Mg}, \mathrm{Si}$, and $\mathrm{Ti}$ abundances (from our determinations where available; otherwise from literature sources described in Section 2) for lines with significant blending with these elements. We have also assumed $[\mathrm{C} / \mathrm{Fe}]$, $[\mathrm{N} / \mathrm{Fe}]$, and $[\mathrm{O} / \mathrm{Fe}]=-0.20,+0.20$, and 0.00 dex, respectively, and a $\mathrm{C}^{12} / \mathrm{C}^{13}$ ratio of 10 for our sample stars, based on typical red clump star abundances from Tautvaišienè et al. (2010). Smoothing for each instrument and wavelength region was set by carefully fitting un- 
Table 4. Atmospheric Parameters of Newly Analyzed Stars

\begin{tabular}{|c|c|c|c|c|c|c|c|c|c|c|c|}
\hline Cluster & $\begin{array}{l}\text { Star } \\
\text { I.D. }\end{array}$ & Source $^{\mathrm{b}}$ & $\begin{array}{l}\mathrm{T}_{\text {eff }} \\
(\mathrm{K})\end{array}$ & $\begin{array}{l}\log (g) \\
(\operatorname{dex})\end{array}$ & $\begin{array}{c}\xi \\
\left(\mathrm{km} \mathrm{s}^{-1}\right)\end{array}$ & $\mathrm{FeI}$ & $\begin{array}{l}\sigma \mathrm{FeI} \\
(\mathrm{dex})\end{array}$ & $\begin{array}{l}\text { FeI } \\
\text { no. }\end{array}$ & FeII & $\begin{array}{l}\sigma \mathrm{FeII} \\
(\mathrm{dex})\end{array}$ & $\begin{array}{c}\text { FeII } \\
\text { no. }\end{array}$ \\
\hline Be 31 & 886 & This work & 4500 & 1.8 & 1.05 & 7.17 & 0.12 & 55 & 7.18 & 0.04 & 7 \\
\hline Cr 261 & 1045 & This work & 4500 & 2.0 & 1.15 & 7.49 & 0.13 & 60 & 7.51 & 0.13 & 10 \\
\hline Cr 261 & 1080 & This work & 4500 & 2.1 & 1.10 & 7.44 & 0.13 & 61 & 7.42 & 0.08 & 10 \\
\hline N1817 & 206 & This work & 4750 & 2.1 & 1.35 & 7.45 & 0.12 & 60 & 7.45 & 0.13 & 11 \\
\hline N1817 & 1456 & This work & 4750 & 2.1 & 1.45 & 7.48 & 0.12 & 58 & 7.48 & 0.10 & 11 \\
\hline N2141 & 514 & This work & 4400 & 1.6 & 1.35 & 7.44 & 0.12 & 59 & 7.42 & 0.09 & 11 \\
\hline N2141 & 1821 & This work & 4350 & 1.5 & 1.05 & 7.44 & 0.13 & 59 & 7.44 & 0.12 & 10 \\
\hline N6192 & 9 & This work & 4900 & 1.9 & 1.60 & 7.69 & 0.09 & 53 & 7.69 & 0.11 & 11 \\
\hline N6192 & 45 & This work & 4900 & 2.5 & 1.35 & 7.61 & 0.11 & 55 & 7.62 & 0.06 & 11 \\
\hline N6192 & 96 & This work & 4800 & 1.9 & 1.75 & 7.57 & 0.12 & 52 & 7.58 & 0.07 & 11 \\
\hline N6192 & 137 & This work & 4550 & 1.8 & 1.65 & 7.59 & 0.13 & 47 & 7.61 & 0.09 & 11 \\
\hline N6939 & 53 & This work & 4900 & 2.4 & 1.30 & 7.56 & 0.11 & 59 & 7.56 & 0.12 & 11 \\
\hline N6939 & 65 & This work & 4950 & 2.3 & 1.40 & 7.50 & 0.11 & 57 & 7.49 & 0.10 & 11 \\
\hline N7789 & 212 & This work & 4400 & 1.8 & 1.45 & 7.50 & 0.15 & 56 & 7.50 & 0.17 & 14 \\
\hline N7789 & 468 & This work & 4250 & 1.5 & 1.65 & 7.46 & 0.14 & 54 & 7.48 & 0.15 & 14 \\
\hline N7789 & 605 & This work & 4900 & 2.5 & 1.55 & 7.52 & 0.16 & 58 & 7.53 & 0.11 & 12 \\
\hline N7789 & 765 & This work & 4450 & 2.0 & 1.50 & 7.54 & 0.14 & 57 & 7.55 & 0.13 & 13 \\
\hline N7789 & 958 & This work & 5050 & 2.8 & 1.50 & 7.56 & 0.16 & 59 & 7.55 & 0.10 & 13 \\
\hline Be 31 & 886 & Y05 & 4490 & 1.9 & 1.22 & 6.97 & 0.23 & 35 & 7.05 & 0.18 & 7 \\
\hline Cr 261 & 1045 & $\mathrm{C} 05$ & 4470 & 2.07 & 1.23 & 7.55 & 0.16 & 126 & 7.49 & 0.19 & 10 \\
\hline Cr 261 & 1045 & F03 & 4400 & 1.5 & 1.20 & 7.36 & 0.20 & 37 & 7.31 & 0.18 & 6 \\
\hline Cr 261 & 1080 & $\mathrm{C} 05$ & 4500 & 2.09 & 1.23 & 7.54 & 0.15 & 114 & 7.48 & 0.26 & 11 \\
\hline Cr 261 & 1080 & F03 & 4490 & 2.2 & 1.20 & 7.41 & 0.25 & 41 & 7.45 & 0.22 & 7 \\
\hline N6192 & 9 & M10 & 5050 & 2.3 & 1.75 & 7.66 & 0.07 & 59 & $\ldots$ & $\ldots$ & 8 \\
\hline N6192 & 45 & M10 & 5020 & 2.55 & 1.60 & 7.55 & 0.08 & 119 & $\ldots$ & $\ldots$ & 9 \\
\hline N6192 & 96 & M10 & 5050 & 2.3 & 2.10 & 7.60 & 0.10 & 77 & $\ldots$ & $\ldots$ & 8 \\
\hline N6192 & 137 & M10 & 4670 & 2.1 & 1.80 & 7.54 & 0.08 & 91 & $\ldots$ & $\ldots$ & 9 \\
\hline N6939 & 53 & JFP07 & 4920 & 2.4 & 1.70 & 7.41 & 0.17 & 22 & 7.54 & 0.10 & 7 \\
\hline N6939 & 65 & JFP07 & 5100 & 3.0 & 1.80 & 7.55 & 0.20 & 22 & 7.56 & 0.06 & 8 \\
\hline N7789 & 468 & T05 & 4190 & 1.3 & 1.90 & $7.44^{\mathrm{a}}$ & 0.10 & 22 & $7.44^{\mathrm{a}}$ & 0.03 & 3 \\
\hline N7789 & 765 & T05 & 4430 & 1.8 & 1.70 & $7.48^{\mathrm{a}}$ & 0.09 & 22 & $7.48^{\mathrm{a}}$ & 0.07 & 3 \\
\hline
\end{tabular}

${ }^{a}$ Solar abundances are not given in Tautvaišienè et al. (2005), so we assume $\epsilon\left(\mathrm{Fe}_{\odot}\right)=7.52$

${ }^{b}$ References for atmospheric parameters: C05 = Carretta et al. (2005); F03 = Friel et al. (2003); JFP07 = Jacobson et al. (2007); M10 = Magrini et al. (2010); T05 = Tautvaišienè et al. (2005); Y05 = Yong et al. (2005)

blended surrounding lines within a $10 \AA$ window of the feature of interest.

In the interest of making all the abundances as homogenous as possible, we have employed a $\mathrm{C}$ code to automate the spectral synthesis. It takes a smoothing value, continuum parameter, and set of auxiliary abundances for blends, and calculates synthetic spectra for a range of abundances of the desired element using MOOG. It then compares the observed spectrum in the area of the feature being measured, and selects the best fit to the data based on the $\chi^{2}$ value. In Figure 1 we show a sample europium synthesis fit selected by the program for NGC 6939 star 31; the se- lected best fit abundance is marked with a solid line, and the best fit abundance plus and minus 0.2 dex are marked with dashed and dotted lines, respectively. As we show in Section 6, the error on the Eu abundance for this star due to atmospheric parameter and continuum errors (added in quadrature) is 0.2 dex.

Our automated synthesis program generally does well selecting the best fit. In Figure 2 we show the results of europium measurements from the automated synthesis versus previous europium results from synthesis of the 6437 and $6645 \AA$ lines 'by eye' (Jacobson \& Friel 2013). There is a slight offset between the two measurements: $<[\mathrm{Eu} / \mathrm{Fe}]_{\mathrm{JF} 13}-[\mathrm{Eu} / \mathrm{Fe}]_{\text {this }}>=0.05 \pm 0.10$ dex. In 
Figure 2 the largest positive differences $\left([\mathrm{Eu} / \mathrm{Fe}]_{\mathrm{JF} 13}<\right.$ $\left.[\mathrm{Eu} / \mathrm{Fe}]_{\text {this }}\right)$ are for two relatively high-metallicity clusters (NGC 7142 and NGC 188), which could be explained by differences in the modeling of $\mathrm{CN}$ features or continuum setting. Over the range of the data, the difference from a slope of 1 (dotted line) is smaller than the scatter around the fit, so we conclude that there is no systematic difference between fits by eye and by our automated synthesis program.

As we discuss in detail in later sections, stellar abundance dispersions are typically less than $0.10 \mathrm{dex}$, so the errors in the fits to individual features due to the $\mathrm{S} / \mathrm{N}$ of the spectra should not be significantly larger than this. We discuss the possible effects of blending to various lines in the sections for each element, and the errors due to the continuum setting parameter are discussed in Section 6 .

Linelists used for the synthesis of neutron-capture features were selected from recent literature studies of atomic data for each element. The selection of sources for individual lines is described in more detail in sections 4.1 - 4.6. Atomic data for blends and surrounding lines within the $10 \AA$ fitting window were taken from a comprehensive linelist we have received courtesy of Chris Sneden. All linelists used for synthesis include molecular features. In order to establish the zero point for our abundances, some of which have little data in the literature, we have measured solar and Arcturus abundances for the selected neutron-capture lines using high-resolution and signal-to-noise spectra of Hinkle et al. (2000). For our solar synthesis, we use Anders \& Grevesse (1989) input abundances; for Arcturus, we use CNO abundances from Abia et al. (2012) because these give the best visual fit to molecular features in our linelists, and other elemental abundances are taken from Ramírez \& Allende Prieto (2011). The following sections discuss the solar abundances, hyperfine structure, isotopic ratios, and other atomic data for the individual elements we discuss in this paper. The wavelength and species of lines measured, type and sources of atomic data, and solar and Arcturus abundance measurements for each line are given in Table 5. The Arcturus abundance ratios to $\mathrm{Fe}$ given in the last column are relative to the literature solar values from Anders \& Grevesse (1989), not our measured solar abundances.

\subsection{Europium}

Europium is an almost pure r-process element, with $98 \%$ of solar Eu originating from the r-process. It has two main isotopes, $\mathrm{Eu}^{151}$ and $\mathrm{Eu}^{153}$, which make up 47.8 and $52.2 \%$ of solar europium, respectively (Anders \& Grevesse 1989). Europium lines also display considerable hyperfine structure, necessitating measurement via spectral synthesis. We use the Lawler et al. (2001) linelists with hyperfine and isotopic structure for the four Eu II lines with abundances presented here: $4129 \AA$, $4205 \AA, 6437 \AA$, and $6645 \AA$. Because the KPNO, CTIO, HET, and VLT spectra have a limited wavelength range, and some of the Keck and APO spectra have low $\mathrm{S} / \mathrm{N}$ in the blue, the 4129 and $4205 \AA$ lines were only measured in a subset of the sample stars. Line-by-line abundances for the neutron-capture elements are given in Table 6; stellar average abundances are given in Table 7, as well as the stellar dispersions based on the standard deviations of the abundances given by individual lines.

All four of the europium lines are affected by blending to some extent. We have measured the maximum impact of blends to each line by finding the difference in measured abundances with the blend present and removed from the linelist for the Sun and in Arcturus. The $6645 \AA$ line is the least blended; Lawler et al. (2001) note that small $\mathrm{Cr}$ and $\mathrm{Si}$ lines are present in the solar spectrum, but these make $<0.05$ dex difference in the measured solar and Arcturus abundances, and CNO lines are insignificant in this wavelength region. The $6437 \AA$ line has more significant blending from $\mathrm{CNO}$ and Si lines, particularly the Si I line at $6437.71 \AA$ : this line makes less than 0.10 dex difference to the Arcturus line abundance but makes up the majority of the solar profile. We adopt the Arcturus-based log(gf) for this Si line from Jacobson \& Friel (2013). Eu abundances based on this line do not differ systematically from the $6645 \AA$ line $(<6645-6437>=+0.02 \pm 0.08 \mathrm{dex})$ so we see no reason to exclude it in our final abundances. As we discuss in Section 7, the use of the Eu 6645 line alone instead of the stellar averages in Table 7 has a minimal impact on our conclusions.

The $4129 \AA$ and $4205 \AA$ lines are larger than the lines in the red but are thought to have more severe blending, particularly from $\mathrm{C}_{2}$ and $\mathrm{CN}$ lines. Removing these blends makes about 0.10 dex difference to the final Arcturus abundance and up to 0.25 dex difference to the solar abundance for each line. Including these large blends results in a poor fit to the solar spectrum and a low solar $\mathrm{Eu}$ abundance. We therefore removed the $\mathrm{CN}$ and $\mathrm{C}_{2}$ blends from our linelists for synthesis of these two lines. The $4205 \AA$ line also has a significant V blend.

We find an average $\epsilon_{\odot}(\mathrm{Eu})$ of $0.52 \pm 0.03 \mathrm{dex}$ and an Arcturus $[\mathrm{Eu} / \mathrm{Fe}]$ of $0.30 \pm 0.05$ dex (normalized to literature solar abundances). Our solar abundance matches the Anders \& Grevesse (1989) value (0.51) and the Lawler et al. (2001) value (0.52) very well. A [Eu/Fe] ratio of +0.30 puts Arcturus within the range of thick disk $\mathrm{Eu}$ abundances for $[\mathrm{Fe} / \mathrm{H}] \sim-0.50$ from Reddy et al. (2006). When normalized to our solar abundance scale, the Arcturus $[\mathrm{Eu} / \mathrm{Fe}]=0.29 \pm 0.01$; our solar and Arcturus abundances for the individual lines scale well. 


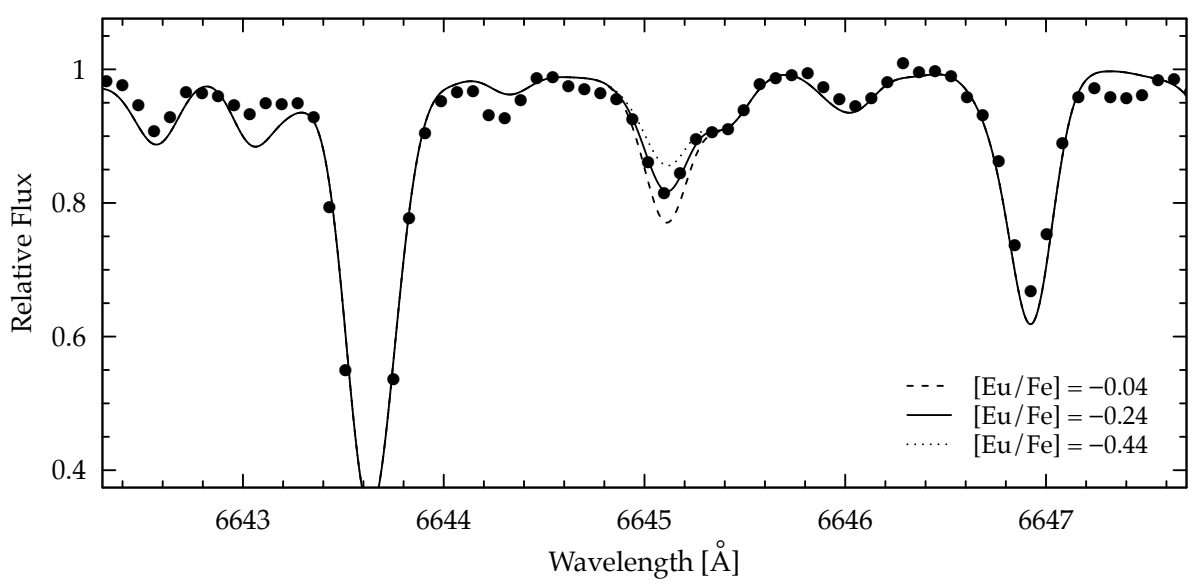

Figure 1. Example fits to the $6645 \AA$ europium line in NGC 6939 star 31 . Dots are the observed spectrum, the solid line represents the selected best fit, and the dashed and dotted lines are the best fit abundance $+/-0.2$ dex, respectively.

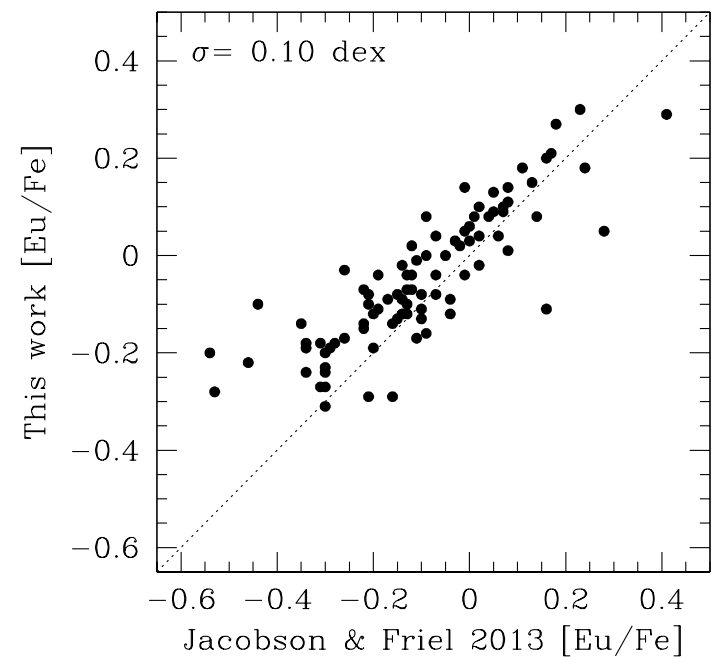

Figure 2. Comparison between $[\mathrm{Eu} / \mathrm{Fe}]$ values for the 6437 and $6645 \AA$ lines as measured by Jacobson \& Friel (2013) and this work. The dashed line represents a 1:1 correspondence.

\subsection{Gadolinium}

Gadolinium is another primarily r-process element, with $82 \%$ of solar gadolinium originating in the r-process (Sneden et al. 2008). It is comprised of seven main isotopes, $\mathrm{Gd}^{152}, \mathrm{Gd}^{154}, \mathrm{Gd}^{155}, \mathrm{Gd}^{156}, \mathrm{Gd}^{157}, \mathrm{Gd}^{158}$, and $\mathrm{Gd}^{160}$, making up $0.3,2.7,14.2,20.7,15.7,25.1$, and $21.3 \%$ of solar Gd, respectively. The hyperfine and isotopic structure of gadolinium have not yet been sufficiently resolved to be taken into account here, so we use single-line atomic data from Den Hartog et al. (2006).

Unfortunately, there are no strong gadolinium lines within the wavelength range of our KPNO spectra, so we were only able to measure Gd abundances in the Keck, APO, and McDonald $2.7 \mathrm{~m}$ spectra. Some of the lines in the solar spectrum measured by Den Hartog et al. (2006) are also impossible to measure given the resolution and signal-to-noise of our data, but we have isolated a set of five lines that we deem trustworthy in our spectra. The 4085, 4191, 4316, 4483, and $4498 \AA \mathrm{Gd}$ II lines are strong and clean enough to give reasonable abundances; the median dispersion on these lines for a single star is 0.08 dex.

There are two lines, 4316 and $4498 \AA$, that have unaccounted for blending in the solar spectrum, but since the measured Arcturus abundances for all Gd lines are similar $(\epsilon \sim 0.40)$, we have included these lines assuming solar abundances from Anders \& Grevesse (1989). In Figure 3 we show a sample gadolinium synthesis fit for the $4316 \AA$ line for Be 32 star 18 . The other three lines included have solar abundances very similar to Anders $\&$ Grevesse (1989) $\epsilon=1.12$. Our solar measurement for the $4191 \AA$ line is 0.12 dex higher than Den Hartog et al. (2006), but this difference may be due to continuum placement as the line is weak in the solar spectrum. Our solar abundance based on all five lines is $\epsilon_{\odot}=1.14$ $\pm 0.02 \mathrm{dex}$, and our Arcturus $[\mathrm{Gd} / \mathrm{Fe}]=0.40 \pm 0.05$ dex.

Again, line-by-line abundances for the for the 20 stars in 9 clusters with blue-extended spectra are in Table 6, and stellar average abundances and dispersions based on the standard deviations of line abundances are given in Table 7. Due to the wavelength region they occupy, these $\mathrm{Gd}$ lines are blended with $\mathrm{CN} / \mathrm{C}_{2}$ and Fe features. The strength of the $\mathrm{CN}$ blending ranges from a maximum 0.15 dex increase in the measured Arcturus abundance in the $4085 \AA$ line to no discernible effect on the $4316 \AA$ line. All lines except for $4498 \AA$ also appear to have some Fe blending.

\subsection{Dysprosium}

Dysprosium is another primarily r-process element, with $88 \%$ of solar dysprosium originating in the $r$ process. It has five primary isotopes, ${ }^{160} \mathrm{Dy},{ }^{161} \mathrm{Dy}$, ${ }^{162}$ Dy, ${ }^{163}$ Dy and ${ }^{164}$ Dy, which make up $2.2 \%, 19.3 \%$, 
Table 5. Solar and Arcturus Abundances

\begin{tabular}{|c|c|c|c|c|c|c|c|c|c|}
\hline $\begin{array}{c}\lambda \\
{[\AA]}\end{array}$ & Species & Structure? & E.P. & $\begin{array}{c}\log (g f)^{a} \\
(\operatorname{dex})\end{array}$ & Source $^{\mathrm{b}}$ & $\begin{array}{c}\text { A\&G89 } \\
\epsilon_{\odot}\end{array}$ & $\begin{array}{c}\text { Our } \\
\epsilon_{\odot}\end{array}$ & $\begin{array}{l}\text { Our } \\
\epsilon_{\text {Arc }}\end{array}$ & $\begin{array}{c}\text { Arc. } \\
{[\mathrm{X} / \mathrm{Fe}]^{\mathrm{c}}}\end{array}$ \\
\hline 5570.44 & Mo I & $\ldots$ & 1.335 & -0.34 & WB88 & 1.92 & $1.92^{\mathrm{d}}$ & 1.58 & 0.19 \\
\hline 5751.41 & Mo I & $\ldots$ & 1.420 & -1.01 & WB88 & 1.92 & $1.92^{\mathrm{d}}$ & 1.53 & 0.14 \\
\hline 6030.64 & Mo I & $\ldots$ & 1.531 & -0.52 & WB88 & 1.92 & 1.91 & 1.55 & 0.16 \\
\hline 5219.04 & Pr II & hfs & 0.795 & -0.05 & S09 & 0.71 & 0.66 & 0.18 & 0.00 \\
\hline 5259.72 & Pr II & hfs & 0.633 & 0.11 & S09 & 0.71 & 0.71 & 0.22 & 0.04 \\
\hline 5322.77 & Pr II & hfs & 0.482 & -0.12 & S09 & 0.71 & 0.63 & 0.14 & -0.04 \\
\hline 5352.40 & Pr II & hfs & 0.482 & -0.74 & S09 & 0.71 & 0.85 & 0.20 & 0.02 \\
\hline 5132.33 & Nd II & $\ldots$ & 0.559 & -0.71 & DH03 & 1.50 & $1.50^{\mathrm{d}}$ & 1.03 & 0.06 \\
\hline 5306.46 & $\mathrm{Nd}$ II & $\ldots$ & 0.859 & -0.97 & DH03 & 1.50 & 1.57 & 1.04 & 0.07 \\
\hline 5356.96 & $\mathrm{Nd}$ II & $\ldots$ & 1.263 & -0.28 & DH03 & 1.50 & 1.46 & 0.94 & -0.03 \\
\hline 5416.37 & $\mathrm{Nd}$ II & $\ldots$ & 0.859 & -0.93 & M75 & 1.50 & 1.42 & 0.89 & -0.08 \\
\hline 5431.52 & $\mathrm{Nd}$ II & $\ldots$ & 1.120 & -0.47 & DH03 & 1.50 & 1.59 & 1.01 & 0.04 \\
\hline 5485.70 & Nd II & $\ldots$ & 1.263 & -0.12 & DH03 & 1.50 & 1.61 & 1.01 & 0.04 \\
\hline 5533.82 & Nd II & $\ldots$ & 0.559 & -1.23 & DH03 & 1.50 & 1.41 & 0.77 & -0.20 \\
\hline 5740.86 & $\mathrm{Nd}$ II & $\ldots$ & 1.159 & -0.53 & DH03 & 1.50 & 1.46 & 0.95 & -0.02 \\
\hline 5811.57 & $\mathrm{Nd}$ II & $\ldots$ & 0.859 & -0.86 & DH03 & 1.50 & 1.43 & 0.90 & -0.07 \\
\hline 6385.15 & $\mathrm{Nd}$ II & $\ldots$ & 1.160 & -0.77 & M75 & 1.50 & 1.41 & 0.87 & -0.10 \\
\hline 4129.72 & Eu II & $\mathrm{hfs} /$ iso & 0.000 & 0.22 & L01 & 0.51 & 0.50 & 0.27 & 0.29 \\
\hline 4205.04 & Eu II & $\mathrm{hfs} /$ iso & 0.000 & 0.21 & L01 & 0.51 & 0.56 & 0.32 & 0.34 \\
\hline 6437.64 & $\mathrm{Eu} \mathrm{II}$ & $\mathrm{hfs} /$ iso & 1.319 & -0.32 & L01 & 0.51 & 0.51 & 0.26 & 0.28 \\
\hline 6645.10 & Eu II & $\mathrm{hfs} /$ iso & 1.379 & 0.12 & L01 & 0.51 & 0.49 & 0.25 & 0.27 \\
\hline 4085.56 & Gd II & $\ldots$ & 0.731 & -0.01 & DH06 & 1.12 & 1.15 & 0.97 & 0.38 \\
\hline 4191.08 & Gd II & $\ldots$ & 0.427 & -0.48 & DH06 & 1.12 & 1.14 & 1.07 & 0.48 \\
\hline 4316.05 & Gd II & $\ldots$ & 0.662 & -0.45 & DH06 & 1.12 & $1.12^{\mathrm{d}}$ & 0.97 & 0.38 \\
\hline 4483.33 & Gd II & $\ldots$ & 1.059 & -0.42 & DH06 & 1.12 & 1.17 & 0.96 & 0.37 \\
\hline 4498.29 & Gd II & $\ldots$ & 0.427 & -1.08 & DH06 & 1.12 & $1.12^{\mathrm{d}}$ & 0.96 & 0.37 \\
\hline 4073.12 & Dy II & $\ldots$ & 0.538 & -0.32 & S09 & 1.10 & 1.17 & 0.97 & 0.40 \\
\hline 4449.70 & Dy II & $\ldots$ & 0.000 & -1.03 & S09 & 1.10 & 1.10 & 0.98 & 0.41 \\
\hline 5169.69 & Dy II & $\ldots$ & 0.103 & -1.95 & S09 & 1.10 & 1.10 & 0.92 & 0.35 \\
\hline
\end{tabular}

${ }^{a}$ For features with hyperfine and/or isotopic structure, the value given is the total $\log (\mathrm{gf})$ for all lines

${ }^{b}$ Sources: WB88 = Whaling \& Brault (1988), S09 = Sneden et al. (2009), DH03 = Den Hartog et al.

(2003), M75 = Meggers et al. (1975), L01 = Lawler et al. (2001), DH06 = Den Hartog et al. (2006)

${ }^{c}$ Relative to literature solar values from Anders \& Grevesse (1989)

$d_{\text {Anders \& Grevesse (1989) solar abundances are assumed for this line (see text) }}$

$28.6 \%, 23.2 \%$ and $26.7 \%$ of solar Dy, respectively (Sneden et al. 2008). Sneden et al. (2009) have noted that while Dy lines should have both isotopic and hyperfine structure, the contributions of these features to the line widths are small and may be ignored to fit the line as a single feature. We follow their lead in adopting the Wickliffe et al. (2000) $\log (\mathrm{gf})$ values.

Sneden et al. (2009) identified 8 lines in the sun that fall within the wavelength range of our Keck, APO, and McDonald $2.7 \mathrm{~m}$ data, one of which could also be measured in the KPNO spectra. This Dy II line at $5169.69 \AA$ is unfortunately weak and heavily blended, and yields consistent results only for our highest-resolution spectra. Our measurements are again limited to our high- resolution/blue-extended spectra for the strongest and least-blended Dy II lines at $4073 \AA$ and $4449 \AA$, and the $5169.69 \AA$ line. In Figure 4 we show a sample dysprosium synthesis fit for the $4449 \AA$ line selected by the program for Be 32 star 18 . We have decided not to include the $4077 \AA$ line, which is the most commonly measured in Dy abundance studies, because it sits on top of the $\mathrm{Sr}$ $4077 \AA$ resonance line which is very strong in our OC stars.

Our solar Dy abundance based on the three lines selected is $1.12 \pm 0.04$, very similar to Anders \& Grevesse (1989) $\epsilon_{\odot}=1.10$. The individual solar abundances for the first two lines match Sneden et al. (2009), but they were not able to measure the $5169 \AA$ line in the sun, pos- 
Table 6. Line-by-Line Abundances ${ }^{\mathrm{a}}$

\begin{tabular}{|c|c|c|c|c|c|c|c|c|c|c|}
\hline Cluster & Star & $\begin{array}{c}4129 \\
{[\mathrm{Eu} / \mathrm{Fe}]}\end{array}$ & $\begin{array}{c}4205 \\
{[\mathrm{Eu} / \mathrm{Fe}]}\end{array}$ & $\begin{array}{c}6437 \\
{[\mathrm{Eu} / \mathrm{Fe}]}\end{array}$ & $\begin{array}{c}6645 \\
{[\mathrm{Eu} / \mathrm{Fe}]}\end{array}$ & $\begin{array}{c}4085 \\
{[\mathrm{Gd} / \mathrm{Fe}]}\end{array}$ & $\begin{array}{c}4191 \\
{[\mathrm{Gd} / \mathrm{Fe}]}\end{array}$ & $\begin{array}{c}4316 \\
{[\mathrm{Gd} / \mathrm{Fe}]}\end{array}$ & $\begin{array}{c}4483 \\
{[\mathrm{Gd} / \mathrm{Fe}]}\end{array}$ & $\begin{array}{c}4498 \\
{[\mathrm{Gd} / \mathrm{Fe}]}\end{array}$ \\
\hline Be17 & 265 & $\ldots$ & $\ldots$ & 0.04 & 0.10 & $\ldots$ & $\ldots$ & $\ldots$ & $\cdots$ & $\ldots$ \\
\hline Be17 & 569 & $\ldots$ & $\ldots$ & -0.13 & -0.08 & $\ldots$ & $\ldots$ & $\ldots$ & $\ldots$ & $\ldots$ \\
\hline Be17 & 1035 & $\ldots$ & $\ldots$ & -0.02 & 0.03 & $\ldots$ & $\ldots$ & $\ldots$ & $\ldots$ & $\ldots$ \\
\hline Be18 & 1163 & -0.02 & -0.04 & 0.18 & 0.21 & 0.08 & 0.19 & $\ldots$ & 0.23 & 0.37 \\
\hline Be18 & 1383 & 0.10 & 0.13 & $\ldots$ & 0.20 & 0.20 & 0.17 & 0.22 & 0.30 & 0.26 \\
\hline $\mathrm{Be} 21$ & 50 & -0.02 & -0.06 & 0.04 & 0.02 & $\ldots$ & 0.37 & 0.33 & .. & 0.26 \\
\hline $\mathrm{Be} 21$ & 51 & $\ldots$ & $\ldots$ & 0.08 & 0.09 & $\ldots$ & 0.28 & 0.24 & 0.2 & 0.31 \\
\hline $\mathrm{Be} 22$ & 414 & $\ldots$ & $\ldots$ & 0.3 & 0.27 & $\ldots$ & 0.18 & 0.34 & $\ldots$ & 0.28 \\
\hline $\mathrm{Be} 22$ & 643 & $\ldots$ & $\ldots$ & 0.29 & 0.18 & $\ldots$ & 0.23 & 0.09 & 0.23 & 0.23 \\
\hline
\end{tabular}

$a_{\text {Table }} 6$ is published in its entirety in the electronic edition of the AJ. A portion is shown here for guidance regarding its form and content.

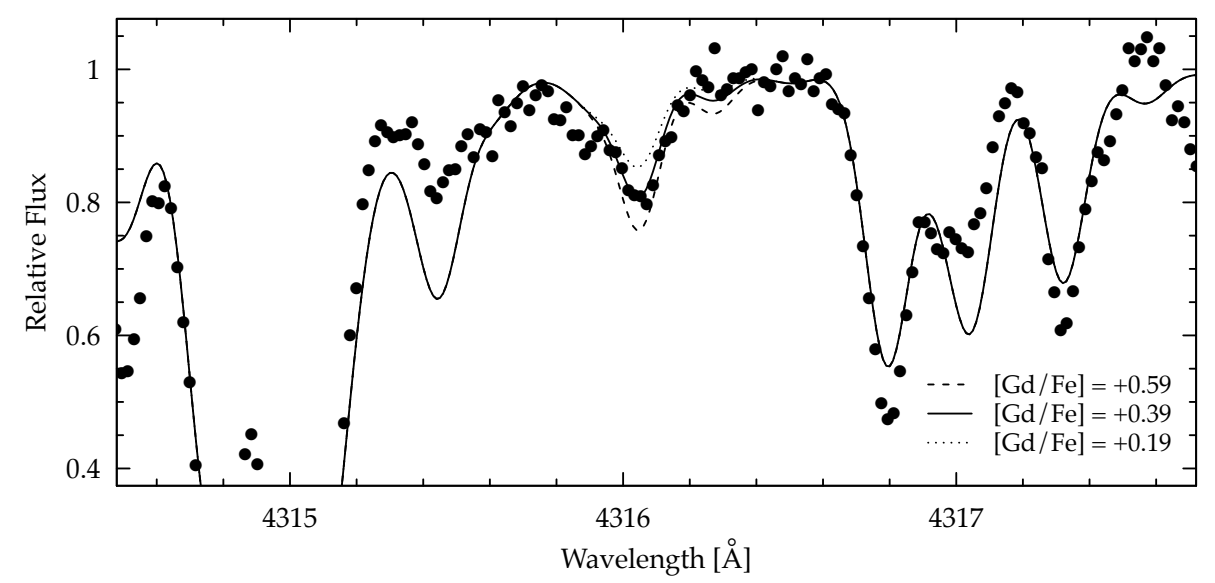

Figure 3. Example fits to the $4316 \AA$ gadolinium line in Be 32 star 18. Dots are the observed spectrum, the solid line represents the selected best fit, and the dashed and dotted lines are the best fit abundance $+/-0.2$ dex, respectively.

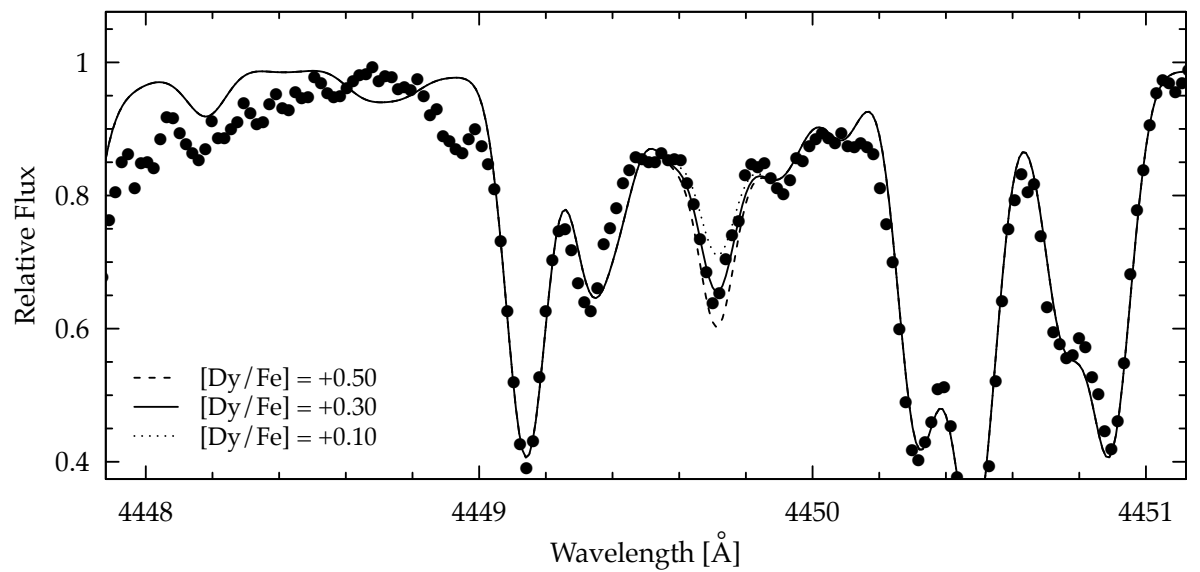

Figure 4. Example fits to the $4449 \AA$ dysprosium line in Be 32 star 18 . Dots are the observed spectrum, the solid line represents the selected best fit, and the dashed and dotted lines are the best fit abundance $+/-0.2$ dex, respectively. 
sibly due to issues with blending. Our Arcturus [Dy/Fe] $=0.39 \pm 0.03$, an enhancement similar to the $[\mathrm{Eu} / \mathrm{Fe}]$ and $[\mathrm{Gd} / \mathrm{Fe}]$ abundances.

The $4073 \AA$ line has significant $\mathrm{CN}$ blending that affects the measured Arcturus abundance by up to 0.15 dex. The $4449 \AA$ line is blended with a Pr feature at $4449.83 \AA$ for which hyperfine structure data is included in Sneden et al. (2009) (see Section 4.5). The 5169 line has a Ti blend listed at $5169.701 \AA$ in our linelist source, but including the Ti blend gives discrepant solar and Arcturus abundances so we do not include it in our final linelist. The median dispersion on our stellar Dy abundances is 0.07 dex.

\subsection{Molybdenum}

Molybdenum is a mixed neutron-capture element; $32 \%$ of solar Mo was formed via the r-process. It has five primary isotopes, ${ }^{95} \mathrm{Mo},{ }^{96} \mathrm{Mo},{ }^{97} \mathrm{Mo},{ }^{98} \mathrm{Mo}$, and ${ }^{100} \mathrm{Mo}$, which are $20.4,24.1,12.3,30.8$, and $12.3 \%$ of total solar Mo, respectively (Sneden et al. 2008). We only have data for Mo I lines, which are weak and probably do not require hyperfine or isotopic structure. Data for structure constants of Mo are unavailable at this point; there is very little in the literature on the measurement of Mo in stars. We identify trustworthy lines from the GES linelist (Heiter et al. 2015), which takes Mo atomic data from Whaling \& Brault (1988), and model each feature as a single line.

There are six Mo I lines in the GES linelist that are within the wavelength range of our full data set. Three of these $(5506.49,5533.03$, and $5858.27 \AA)$ are quite weak lines with large Fe blends; the other three (5570.44, 5751.41 , and $6030.64 \AA$ ) are stronger and clean enough to measure in our OC spectra. The three lines selected have little $\mathrm{CN}$ blending $(<0.05$ dex change in the Arcturus abundance) and no major blended features.

Two of the three Mo lines, 5570 and $5751 \AA$, are too weak to be measured in our solar spectrum, but measured Arcturus abundances are similar, giving $[\mathrm{Mo} / \mathrm{Fe}]_{\mathrm{Arc}}=0.16 \pm 0.03 \mathrm{dex}$, so we assume these lines have solar abundances similar to the $6030 \AA$ line which matches the Anders \& Grevesse (1989) solar abundance.

\subsection{Praseodymium}

Solar praseodymium is mostly composed of a single isotope, ${ }^{141} \mathrm{Pr}$, that is $50.9 \% \mathrm{r}$-process. Pr lines display broad hyperfine structure that must be accounted for; we use the linelists of Sneden et al. (2009) to model these effects. Several of the lines they measure in the solar spectrum are too weak or blended to measure in our spectra, but we have selected a set of four Pr lines that give consistent results for our sample stars. Be 31 star 886 and NGC 2141 star 1348 are the only spectra limited to wavelengths redward of all four lines, so they have no Pr abundances.

The $5219,5259,5322$, and $5352 \AA$ lines measured here have very little CNO blending. The $5219 \AA$ line has a significant Co blend that composes the majority of the solar feature profile. The $5322 \AA$ and $5352 \AA$ lines have small $\mathrm{Fe}$ blends that make very little difference to measured Arcturus abundances but could affect solar abundances by up to 0.10 dex. Abundances from the $5219 \AA$ line are not systematically different from the other three less blended lines, and the typical stellar dispersion between Pr line abundances is 0.07 dex, so there is no sign that the blend is causing problems with the measurement of the line. We have assumed $[\mathrm{Co} / \mathrm{Fe}]=0.00$ for all OC stars, as most thin disk stars have approximately solar [Co/Fe] ratios (Reddy et al. 2003).

Our Pr solar measurements are somewhat scattered; our average solar abundance is $\epsilon=0.71 \pm 0.10$. This matches the Anders \& Grevesse (1989) solar Pr abundance, although our 5259 and $5322 \AA$ abundances are lower than those given in Sneden et al. (2009). Different $\log (\mathrm{gf})$ values used for blends are probably the cause of this discrepancy. The average Arcturus $[\mathrm{Pr} / \mathrm{Fe}]=0.01$ \pm 0.03 dex.

\subsection{Neodymium}

Solar Nd is comprised of seven different isotopes, ${ }^{142} \mathrm{Nd},{ }^{143} \mathrm{Nd},{ }^{144} \mathrm{Nd},{ }^{145} \mathrm{Nd},{ }^{146} \mathrm{Nd},{ }^{148} \mathrm{Nd}$, and ${ }^{150} \mathrm{Nd}$, which are $27.2,12.2,23.8,8.3,17.2,5.7$ and $5.6 \%$ of the total abundance, respectively (Sneden et al. 2008). Approximately $42.1 \%$ of solar $\mathrm{Nd}$ was created via the r-process, so we classify it as a mixed r- and s- element. Nd II has many observable lines in optical wavelengths that display minimal hyperfine and isotopic structure with $\Delta \lambda<0.005 \AA$ (Den Hartog et al. 2003). Because isotopic and hyperfine structure data for these features are not generally available, and the resolution of our spectra are significantly lower than the expected wavelength deviations, we do not model this structure with synthesis.

We have selected a set of ten Nd II lines that appear relatively unblended in our spectra. Eight of these lines, at $5132.33,5306.46,5356.96,5431.52,5485.70,5533.82$, 5740.86 , and $5811.57 \AA$ have atomic data listed in Den Hartog et al. (2003) that are used in the GES linelist. Two of the lines, at 5416.37 and $6385.15 \AA$, do not appear in Den Hartog et al. (2003) but are included in the GES linelist with atomic data taken from Meggers et al. (1975). The $5356.96 \AA$ line is partially blended with a Sc II line at $5357.20 \AA$ at the resolution of our KPNO $4 \mathrm{~m}$ spectra, but the features are separated enough to identify that a solar $[\mathrm{Sc} / \mathrm{Fe}]$ ratio fits the $\mathrm{Sc}$ line well for most stars, so we include these measurements in our final data set. The $5533.82 \AA$ line has a significant V I blend at the same wavelength, but assuming solar $[\mathrm{V} / \mathrm{Fe}]$ gives 
abundances in good agreement with the other lines. The median dispersion of the $\mathrm{Nd}$ stellar abundances is 0.08 dex.

We find that $\mathrm{Nd} \epsilon_{\odot}=1.49 \pm 0.08$; this value falls between the Anders \& Grevesse $(1989) \epsilon_{\odot}=1.50$ and Den Hartog et al. (2003) $\epsilon_{\odot}=1.46$. Our average Arcturus $[\mathrm{Nd} / \mathrm{Fe}]=-0.03 \pm 0.09 \mathrm{dex}$, but normalizing to our solar $\mathrm{Nd}$ abundances instead of the literature decreases the dispersion to 0.05 dex; our solar and Arcturus line abundances mostly track each other except for $\lambda 5533$.

\section{DISCREPANT STARS}

We have a few stars in our sample with abundances far from the cluster averages for some or all elements. Calculating cluster abundances including these stars would greatly enhance the cluster dispersions, so in some cases we have excluded them (if we have enough stars in the cluster to determine the outlier). We discuss them here.

NGC 2355: This cluster has a relatively high dispersion for all of the elements, including iron; the cluster $[\mathrm{Fe} / \mathrm{H}]$ dispersion is 0.10 dex, while the median for our cluster sample is 0.03 dex. With only three stars, it is not completely clear which of the three, if any, is distorting the cluster average. Star 144 is +0.20 dex enhanced from the other two in Mo and Nd, but less separated in $\operatorname{Pr}$ and Eu. We will analyze more stars in this cluster in our forthcoming s-process focused paper, and will be able to better define the dispersions for this cluster.

NGC 2141: We have four stars for this cluster, two of which are newly presented in this work. Three of these stars cluster nicely, with dispersions $<0.05$ dex for all elements except Pr, but star 1348 has abundances $\sim 0.40$ dex larger than the others for $\mathrm{Mo}$ and $\mathrm{Nd}$ (we could not measure Pr in this star due to wavelength coverage restrictions). Jacobson \& Friel (2013) also note that this star has enhanced $\mathrm{Zr}, \mathrm{Ba}$, and La compared to star 1007, although its Eu abundances from Jacobson \& Friel (2013) and this paper are not significantly higher than the other cluster members; the additional two stars (514 and 1821) allow us to confirm that 1348 is the anomalous member. We exclude this star when calculating all cluster abundances and dispersions; its enhancement in Mo and Nd, and Eu abundance within $1 \sigma$ of the cluster average, suggest that this is an s-process enhanced star.

NGC 6939: We have six stars for this cluster, including two from our most recent APO run. Star 190 gives enhanced abundances for all of the elements we present here, sometimes by as much as 0.5 dex. Star 121 has slightly lower abundances than the other four, though the difference is not as significant. We have excluded 190 from the final cluster abundance calculations as we have multiple stars for this cluster with more reliable abundances.

\section{ERRORS}

Errors in neutron capture abundances due to errors in atmospheric and continuum setting parameters for six stars from our sample are given in Table 8. These stars encompass the full range of temperatures, gravities, microturbulent velocities, and metallicities of our sample. The values for each element were calculated by averaging the changes in all lines that were measured for each star. The changes in abundance due to changes in the continuum setting parameter are based on a two percent shift in the chosen continuum for each star. Error due to the signal-to-noise of each spectrum and the atomic data used are not contained in these values.

Unfortunately, our Mo lines appear to be relatively sensitive to temperature, changing abundances by $\sim 0.15$ dex per 100K for all but the coolest star (N6939 31). As this is an element that has few stellar abundance measurements in the literature, we must interpret abundances for this element with caution. Dysprosium also appears to be sensitive to temperature; the Dy lines blueward of $5000 \AA$ are fairly strong, but somewhat blended. Abundance errors due to changes in $\log (\mathrm{g})$ are fairly constant for the different stars and elements at around 0.10 dex, except for Mo which is relatively unaffected by changes in $\log (\mathrm{g})$. Changes in the microturbulent velocity have little effect on most stars; Dy experiences the largest effects, which are still less than 0.1 dex. The atmospheric parameter errors added in quadrature are $\sim 0.10$ dex in $\mathrm{Nd}, \mathrm{Pr}$, and $\mathrm{Eu}$; 0.15 dex in Mo and Gd; and 0.20 dex in Dy.

As mentioned in Section 3, we adopt errors of $\pm 100 \mathrm{~K}$, 0.2 dex, and $0.2 \mathrm{~km} \mathrm{~s}^{-1}$ in effective temperature, $\log (\mathrm{g})$, and microturbulent velocity, respectively, to account for both internal errors and differences in methods from study to study. If we were to consider only internal errors of $\pm 75 \mathrm{~K}, 0.1 \mathrm{dex}$, and $0.1 \mathrm{~km} \mathrm{~s}^{-1}$, we would be looking at negligible errors in abundance due to microturbulent velocities for most elements, and no more than 0.05 dex for Dy; abundance errors due to $\log (\mathrm{g})$ would also be on the order of 0.05 dex. Abundance errors due to temperature errors would remain significant, on the order of 0.1 dex for Mo and Dy. The atmospheric parameter errors added in quadrature in this case would be 0.06 dex for $\mathrm{Pr}, \mathrm{Nd}, \mathrm{Eu}$, and Gd; 0.11 dex for Mo; and 0.14 dex for Dy. However, this moderate decrease in atmospheric parameter errors would not significantly affect conclusions presented here as the largest contributions to abundance errors are due to errors in setting the continuum during synthesis fitting.

Running synthesis in a semi-automated way makes the determination of continuum errors more objective than it might otherwise be, and due to the size of the lines being measured (especially Mo) we expect these errors 
to be large. They are $0.10-0.15$ dex for the coolest stars of the six at 4000 and $4400 \mathrm{~K}$, and rise to $0.2-0.3 \mathrm{dex}$ for the hottest stars in our sample at 5200 and $5300 \mathrm{~K}$. The choice of the continuum uncertainty does of course depend on the signal-to-noise of the individual spectra, but for a signal-to-noise per pixel of 100 the rms dispersion of the noise is about 0.01 , so our chosen continuum error is about two times the noise for a typical sample star.

Table 7. Average Stellar Abundances

\begin{tabular}{|c|c|c|c|c|c|c|c|c|c|c|c|c|c|}
\hline Cluster & Star & $\begin{array}{c}\text { Avg. } \\
{[\mathrm{Eu} / \mathrm{Fe}]}\end{array}$ & $\begin{array}{c}\sigma \\
{[\mathrm{Eu} / \mathrm{Fe}]}\end{array}$ & $\begin{array}{c}\text { Avg. } \\
{[\mathrm{Gd} / \mathrm{Fe}]}\end{array}$ & $\begin{array}{c}\sigma \\
{[\mathrm{Gd} / \mathrm{Fe}]}\end{array}$ & $\begin{array}{c}\text { Avg. } \\
{[\mathrm{Dy} / \mathrm{Fe}]}\end{array}$ & $\begin{array}{c}\sigma \\
{[\mathrm{Dy} / \mathrm{Fe}]}\end{array}$ & $\begin{array}{c}\text { Avg. } \\
{[\mathrm{Mo} / \mathrm{Fe}]}\end{array}$ & $\begin{array}{c}\sigma \\
{[\mathrm{Mo} / \mathrm{Fe}]}\end{array}$ & $\begin{array}{c}\text { Avg. } \\
{[\mathrm{Pr} / \mathrm{Fe}]}\end{array}$ & $\begin{array}{c}\sigma \\
{[\mathrm{Pr} / \mathrm{Fe}]}\end{array}$ & $\begin{array}{c}\text { Avg. } \\
{[\mathrm{Nd} / \mathrm{Fe}]}\end{array}$ & $\begin{array}{c}\sigma \\
{[\mathrm{Nd} / \mathrm{Fe}]}\end{array}$ \\
\hline Be17 & 265 & 0.07 & 0.04 & $\ldots$ & $\ldots$ & $\ldots$ & $\ldots$ & 0.01 & 0.03 & -0.10 & 0.06 & 0.01 & 0.12 \\
\hline Be17 & 569 & -0.11 & 0.04 & $\ldots$ & $\ldots$ & $\ldots$ & $\ldots$ & -0.07 & 0.07 & -0.20 & 0.11 & -0.11 & 0.06 \\
\hline Be17 & 1035 & 0.01 & 0.04 & $\ldots$ & $\ldots$ & $\ldots$ & $\ldots$ & -0.05 & 0.07 & -0.09 & 0.07 & 0.03 & 0.11 \\
\hline Be18 & 1163 & 0.08 & 0.13 & 0.22 & 0.12 & 0.24 & 0.04 & 0.13 & 0.08 & 0.18 & 0.02 & 0.22 & 0.07 \\
\hline Be18 & 1383 & 0.14 & 0.05 & 0.23 & 0.05 & 0.23 & 0.03 & 0.05 & 0.05 & 0.21 & 0.09 & 0.19 & 0.07 \\
\hline $\mathrm{Be} 21$ & 50 & -0.01 & 0.04 & 0.32 & 0.06 & 0.15 & 0.18 & 0.17 & 0.08 & 0.18 & 0.05 & 0.21 & 0.05 \\
\hline $\mathrm{Be} 21$ & 51 & 0.09 & 0.01 & 0.26 & 0.05 & 0.19 & 0.03 & 0.09 & 0.10 & 0.19 & 0.03 & 0.19 & 0.05 \\
\hline $\mathrm{Be} 22$ & 414 & 0.29 & 0.02 & 0.27 & 0.08 & 0.31 & 0.16 & 0.06 & 0.05 & 0.22 & 0.02 & 0.32 & 0.04 \\
\hline $\mathrm{Be} 22$ & 643 & 0.24 & 0.08 & 0.20 & 0.07 & 0.21 & 0.06 & 0.18 & 0.06 & 0.32 & 0.17 & 0.41 & 0.10 \\
\hline Be31 & 886 & 0.27 & 0.07 & $\ldots$ & $\ldots$ & $\ldots$ & $\ldots$ & 0.46 & 0.17 & $\ldots$ & $\ldots$ & 0.56 & 0.08 \\
\hline Be32 & 2 & 0.00 & 0.03 & $\ldots$ & $\ldots$ & $\ldots$ & $\ldots$ & 0.01 & 0.05 & -0.11 & 0.10 & -0.03 & 0.04 \\
\hline Be32 & 4 & 0.06 & 0.04 & $\ldots$ & $\ldots$ & $\ldots$ & $\ldots$ & -0.01 & 0.05 & -0.12 & 0.05 & -0.04 & 0.04 \\
\hline Be32 & 16 & 0.11 & 0.03 & 0.34 & 0.08 & 0.41 & 0.06 & 0.15 & 0.01 & 0.15 & 0.03 & 0.15 & 0.05 \\
\hline Be32 & 18 & 0.15 & 0.04 & 0.29 & 0.11 & 0.32 & 0.10 & 0.11 & 0.01 & 0.11 & 0.03 & 0.16 & 0.05 \\
\hline Be39 & 3 & -0.02 & 0.02 & $\ldots$ & $\ldots$ & $\ldots$ & $\ldots$ & 0.01 & 0.02 & -0.01 & 0.10 & 0.04 & 0.07 \\
\hline Be39 & 5 & -0.01 & 0.09 & $\ldots$ & $\ldots$ & $\ldots$ & $\ldots$ & 0.07 & 0.06 & -0.01 & 0.11 & 0.05 & 0.06 \\
\hline Be39 & 12 & 0.08 & 0.00 & $\ldots$ & $\ldots$ & $\ldots$ & $\ldots$ & 0.20 & 0.05 & 0.14 & 0.06 & 0.03 & 0.09 \\
\hline Be39 & 14 & 0.10 & 0.06 & $\ldots$ & $\ldots$ & $\ldots$ & $\ldots$ & 0.16 & 0.01 & 0.14 & 0.08 & 0.11 & 0.08 \\
\hline Cr261 & 1045 & -0.12 & 0.06 & $\ldots$ & $\ldots$ & $\ldots$ & $\ldots$ & -0.08 & 0.07 & -0.30 & $\ldots$ & -0.10 & 0.05 \\
\hline Cr261 & 1080 & -0.03 & 0.01 & $\ldots$ & $\ldots$ & $\ldots$ & $\ldots$ & -0.02 & 0.07 & -0.29 & $\ldots$ & -0.10 & 0.05 \\
\hline M67 & 105 & -0.10 & 0.03 & $\ldots$ & $\ldots$ & $\ldots$ & $\ldots$ & 0.07 & 0.03 & -0.06 & 0.03 & 0.02 & 0.04 \\
\hline M67 & 141 & -0.07 & 0.09 & $\ldots$ & $\ldots$ & $\ldots$ & $\ldots$ & 0.07 & 0.04 & -0.05 & 0.05 & -0.01 & 0.12 \\
\hline M67 & 170 & -0.15 & 0.04 & $\ldots$ & $\ldots$ & $\ldots$ & $\ldots$ & 0.01 & 0.07 & -0.07 & 0.04 & -0.01 & 0.05 \\
\hline N188 & 532 & -0.03 & 0.06 & $\ldots$ & $\ldots$ & $\ldots$ & $\ldots$ & 0.38 & 0.08 & 0.00 & 0.12 & 0.19 & 0.06 \\
\hline N188 & 747 & -0.11 & 0.01 & $\ldots$ & $\ldots$ & $\ldots$ & $\ldots$ & 0.17 & 0.06 & -0.08 & 0.06 & 0.08 & 0.11 \\
\hline N188 & 919 & -0.19 & 0.13 & $\ldots$ & $\ldots$ & $\ldots$ & $\ldots$ & 0.15 & 0.13 & -0.07 & 0.10 & 0.11 & 0.07 \\
\hline N188 & 1224 & -0.15 & 0.05 & $\ldots$ & $\ldots$ & $\ldots$ & $\ldots$ & 0.23 & 0.04 & -0.03 & 0.04 & 0.17 & 0.08 \\
\hline N1193 & 282 & -0.03 & 0.09 & $\ldots$ & $\ldots$ & $\ldots$ & $\ldots$ & 0.28 & 0.08 & 0.14 & 0.10 & 0.05 & 0.07 \\
\hline $\mathrm{N} 1245$ & 10 & -0.26 & 0.02 & $\ldots$ & $\ldots$ & $\ldots$ & $\ldots$ & 0.21 & $\ldots$ & -0.11 & 0.05 & 0.01 & 0.12 \\
\hline N1245 & 125 & -0.09 & 0.05 & $\ldots$ & $\ldots$ & $\ldots$ & $\ldots$ & 0.02 & 0.06 & 0.09 & 0.11 & 0.19 & 0.10 \\
\hline N1245 & 160 & -0.06 & 0.05 & $\ldots$ & $\ldots$ & $\ldots$ & $\ldots$ & 0.09 & $\ldots$ & 0.15 & 0.07 & 0.06 & 0.09 \\
\hline N1245 & 382 & -0.10 & 0.01 & $\ldots$ & $\ldots$ & $\ldots$ & $\ldots$ & 0.15 & $\ldots$ & 0.13 & 0.05 & 0.08 & 0.05 \\
\hline $\mathrm{N} 1817$ & 73 & -0.04 & 0.00 & $\ldots$ & $\ldots$ & $\ldots$ & $\ldots$ & 0.34 & 0.01 & 0.02 & 0.09 & 0.16 & 0.07 \\
\hline $\mathrm{N} 1817$ & 79 & -0.12 & 0.01 & $\ldots$ & $\ldots$ & $\ldots$ & $\ldots$ & 0.22 & 0.13 & 0.13 & 0.06 & 0.14 & 0.06 \\
\hline N1817 & 206 & -0.11 & 0.03 & 0.05 & 0.15 & -0.02 & 0.11 & 0.27 & $\ldots$ & 0.02 & 0.08 & 0.14 & 0.08 \\
\hline N1817 & 1456 & -0.07 & 0.06 & 0.03 & 0.22 & -0.02 & 0.14 & 0.17 & 0.06 & 0.13 & 0.09 & 0.15 & 0.07 \\
\hline N1883 & 8 & -0.22 & 0.02 & $\ldots$ & $\ldots$ & $\ldots$ & $\ldots$ & -0.19 & 0.05 & 0.05 & 0.06 & 0.00 & 0.08 \\
\hline N1883 & 9 & -0.18 & 0.02 & $\ldots$ & $\ldots$ & $\ldots$ & $\ldots$ & 0.11 & 0.16 & 0.03 & 0.12 & 0.03 & 0.10 \\
\hline $\mathrm{N} 2141$ & 514 & -0.16 & 0.01 & 0.02 & 0.10 & $\ldots$ & $\ldots$ & -0.07 & 0.12 & -0.10 & 0.12 & -0.10 & 0.09 \\
\hline $\mathrm{N} 2141$ & 1007 & -0.12 & 0.04 & $\ldots$ & $\ldots$ & $\ldots$ & $\ldots$ & -0.08 & 0.01 & -0.05 & 0.06 & -0.02 & 0.08 \\
\hline $\mathrm{N} 2141$ & 1348 & -0.11 & 0.05 & $\ldots$ & $\ldots$ & $\ldots$ & $\ldots$ & 0.27 & 0.06 & $\ldots$ & $\ldots$ & 0.25 & 0.04 \\
\hline $\mathrm{N} 2141$ & 1821 & -0.21 & $\ldots$ & 0.06 & 0.10 & $\ldots$ & $\ldots$ & -0.13 & 0.10 & -0.29 & 0.10 & -0.08 & 0.10 \\
\hline N2158 & 4230 & -0.23 & 0.08 & $\ldots$ & $\ldots$ & $\ldots$ & $\ldots$ & -0.02 & 0.04 & 0.00 & 0.06 & 0.06 & 0.08 \\
\hline N2194 & 55 & -0.16 & 0.11 & $\ldots$ & $\ldots$ & $\ldots$ & $\ldots$ & 0.21 & 0.11 & 0.10 & 0.06 & 0.25 & 0.09 \\
\hline
\end{tabular}


Table 7 (continued)

\begin{tabular}{|c|c|c|c|c|c|c|c|c|c|c|c|c|c|}
\hline Cluster & Star & $\begin{array}{c}\text { Avg. } \\
{[\mathrm{Eu} / \mathrm{Fe}]}\end{array}$ & $\begin{array}{c}\sigma \\
{[\mathrm{Eu} / \mathrm{Fe}]}\end{array}$ & $\begin{array}{c}\text { Avg. } \\
{[\mathrm{Gd} / \mathrm{Fe}]}\end{array}$ & $\begin{array}{c}\sigma \\
{[\mathrm{Gd} / \mathrm{Fe}]}\end{array}$ & $\begin{array}{c}\text { Avg. } \\
{[\mathrm{Dy} / \mathrm{Fe}]}\end{array}$ & $\begin{array}{c}\sigma \\
{[\mathrm{Dy} / \mathrm{Fe}]}\end{array}$ & $\begin{array}{c}\text { Avg. } \\
{[\mathrm{Mo} / \mathrm{Fe}]}\end{array}$ & $\begin{array}{c}\sigma \\
{[\mathrm{Mo} / \mathrm{Fe}]}\end{array}$ & $\begin{array}{c}\text { Avg. } \\
{[\mathrm{Pr} / \mathrm{Fe}]}\end{array}$ & $\begin{array}{c}\sigma \\
{[\mathrm{Pr} / \mathrm{Fe}]}\end{array}$ & $\begin{array}{c}\text { Avg. } \\
{[\mathrm{Nd} / \mathrm{Fe}]}\end{array}$ & $\begin{array}{c}\sigma \\
{[\mathrm{Nd} / \mathrm{Fe}]}\end{array}$ \\
\hline N2194 & 57 & -0.29 & 0.03 & $\ldots$ & $\ldots$ & $\ldots$ & $\ldots$ & 0.29 & 0.12 & 0.13 & 0.09 & 0.13 & 0.10 \\
\hline N2355 & 144 & 0.02 & 0.05 & $\ldots$ & $\ldots$ & $\ldots$ & $\ldots$ & 0.41 & $\ldots$ & 0.33 & $\ldots$ & 0.36 & 0.08 \\
\hline N2355 & 398 & -0.09 & 0.07 & $\ldots$ & $\ldots$ & $\ldots$ & $\ldots$ & 0.24 & 0.08 & 0.08 & 0.04 & 0.10 & 0.09 \\
\hline N2355 & 668 & -0.12 & 0.04 & $\ldots$ & $\ldots$ & $\ldots$ & $\ldots$ & 0.23 & 0.04 & 0.14 & 0.06 & 0.16 & 0.09 \\
\hline N6192 & 9 & -0.19 & 0.12 & $\ldots$ & $\ldots$ & $\ldots$ & $\ldots$ & -0.06 & 0.02 & -0.14 & 0.06 & -0.10 & 0.05 \\
\hline N6192 & 45 & -0.05 & 0.03 & $\ldots$ & $\ldots$ & $\ldots$ & $\ldots$ & 0.18 & 0.06 & 0.10 & 0.06 & 0.15 & 0.06 \\
\hline N6192 & 96 & -0.15 & 0.05 & $\ldots$ & $\ldots$ & $\ldots$ & $\ldots$ & 0.06 & 0.01 & -0.07 & 0.03 & 0.03 & 0.03 \\
\hline N6192 & 137 & -0.06 & 0.03 & $\ldots$ & $\ldots$ & $\ldots$ & $\ldots$ & 0.02 & 0.01 & -0.03 & 0.06 & 0.08 & 0.06 \\
\hline N6939 & 31 & -0.21 & 0.05 & $\ldots$ & $\ldots$ & $\ldots$ & $\ldots$ & -0.19 & 0.03 & -0.16 & 0.04 & -0.08 & 0.06 \\
\hline N6939 & 53 & -0.31 & 0.05 & -0.01 & 0.15 & -0.10 & 0.12 & 0.10 & 0.07 & 0.02 & 0.11 & 0.01 & 0.10 \\
\hline N6939 & 65 & -0.37 & 0.07 & -0.17 & 0.17 & -0.12 & 0.12 & 0.00 & 0.04 & 0.08 & 0.08 & -0.04 & 0.05 \\
\hline N6939 & 121 & -0.28 & 0.01 & $\ldots$ & $\ldots$ & $\ldots$ & $\ldots$ & -0.38 & 0.06 & -0.27 & 0.08 & -0.26 & 0.07 \\
\hline N6939 & 190 & -0.04 & $\ldots$ & $\ldots$ & $\ldots$ & $\ldots$ & $\ldots$ & 0.41 & 0.08 & 0.33 & 0.08 & 0.49 & 0.05 \\
\hline N6939 & 212 & -0.10 & 0.13 & $\ldots$ & $\ldots$ & $\ldots$ & $\ldots$ & -0.01 & 0.13 & -0.05 & 0.08 & 0.03 & 0.08 \\
\hline N7142 & 196 & -0.12 & 0.11 & $\ldots$ & $\ldots$ & $\ldots$ & $\cdots$ & 0.11 & 0.08 & 0.03 & 0.04 & 0.02 & 0.06 \\
\hline N7142 & 229 & -0.15 & 0.10 & $\ldots$ & $\ldots$ & $\ldots$ & $\ldots$ & 0.15 & 0.05 & -0.06 & 0.09 & 0.01 & 0.06 \\
\hline N7142 & 377 & -0.16 & 0.06 & $\ldots$ & $\ldots$ & $\ldots$ & $\ldots$ & 0.12 & 0.01 & -0.01 & 0.04 & -0.02 & 0.06 \\
\hline N7142 & 421 & -0.14 & 0.06 & $\ldots$ & $\ldots$ & $\ldots$ & $\ldots$ & 0.17 & 0.06 & -0.06 & 0.08 & 0.03 & 0.07 \\
\hline N7789 & 212 & -0.07 & 0.02 & 0.18 & 0.23 & 0.02 & $\ldots$ & 0.17 & 0.04 & 0.07 & 0.05 & 0.15 & 0.06 \\
\hline N7789 & 468 & -0.15 & 0.08 & 0.14 & 0.00 & 0.04 & 0.04 & 0.14 & 0.03 & 0.13 & 0.10 & 0.12 & 0.10 \\
\hline N7789 & 605 & -0.09 & 0.01 & 0.14 & 0.01 & -0.03 & $\ldots$ & 0.20 & 0.18 & 0.05 & 0.06 & 0.17 & 0.09 \\
\hline N7789 & 765 & -0.14 & 0.15 & 0.19 & 0.01 & 0.03 & 0.07 & 0.18 & 0.02 & 0.17 & 0.10 & 0.17 & 0.05 \\
\hline N7789 & 958 & -0.10 & $\ldots$ & 0.19 & 0.01 & 0.11 & 0.00 & 0.43 & $\ldots$ & 0.18 & 0.04 & 0.20 & 0.08 \\
\hline PWM4 & RGB1 & 0.10 & 0.01 & 0.07 & 0.05 & 0.18 & $\ldots$ & -0.06 & 0.04 & 0.12 & 0.09 & 0.14 & 0.06 \\
\hline
\end{tabular}

\section{ABUNDANCE DISCUSSION AND LITERATURE COMPARISON}

\subsection{OC Literature Comparison}

Cluster average abundances are given in Table 9, along with dispersions (the dispersion between stars, or individual features for clusters with one star). There are a few large sets of $\mathrm{OC}$ neutron-capture measurements in the literature, including Yong et al. (2005, 2012), and Reddy et al. (2012, 2013, 2015) which include some overlap with our sample that allows direct comparison. There are also several analyses of smaller numbers of clusters that overlap with our sample; overall, 10 of our 23 sample clusters have Eu abundances in the literature, five of which also have Nd abundances, and one (NGC 7789) that has a Pr abundance. M67 is a particularly well-studied OC with several $\mathrm{Eu}$ measurements in the literature. Table 10 shows relative cluster abundances for elements with literature OC measurements in the sense $\Delta[\mathrm{X} / \mathrm{Fe}]=[\mathrm{X} / \mathrm{Fe}]_{\text {lit. }}-[\mathrm{X} / \mathrm{Fe}]_{\text {our }}$. All abundances in Table 10 have been placed on our abundance scale (including the hyperfine structure correction for Eu abundances as discussed below) except for Tautvaišienė et al. (2000, 2005) which do not list adopted solar abundances.

The neutron-capture elements are the focus of this work, but because $[\mathrm{Fe} / \mathrm{H}]$ abundances form the baseline for our abundance measurements and because many questions still remain about the Galactic $[\mathrm{Fe} / \mathrm{H}]$ gradient (we discuss this further in later sections), we must first consider systematic offsets in our iron abundances. We find that our cluster $[\mathrm{Fe} / \mathrm{H}]$ values are $\sim 0.10$ dex higher than those in the literature, and $\sim 0.15$ dex higher than those found by Yong et al. (2005, 2012). Differences of this magnitude from study to study are not uncommon, and fall within the uncertainty due to atmospheric parameter errors and continuum setting (we adopt uncertainties due to atmospheric parameters for $[\mathrm{Fe} / \mathrm{H}]$ from Jacobson \& Friel (2013) since most $[\mathrm{Fe} / \mathrm{H}]$ cluster measurements were taken from that work). We do not attempt to correct for this relatively small effect but we will consider it further when discussing the neutron-capture literature comparisons.

Because Eu lines display hyperfine and isotopic structure, an abundance comparison for these elements is not as straightforward as equivalent width measurements. 
nation of atmospheric parameter errors (see Table 8), is enough to increase the measured Eu abundance by 0.1 - 0.2 dex.

Generally, literature studies of Eu in OCs rely on measurements of the $6645 \AA$ line alone because it is the least blended of the Eu lines, and we must consider possible effects of the introduction of the other three lines presented here. Our Eu cluster abundances, when calculated only with the $6645 \AA$ line, never differ by more than 0.1 dex from the abundance based on all four lines, with a median difference of 0.03 dex that does not appear to be systematic. We find little difference in trends with Galactocentric radius or age (discussed in the next two sections).

Only one OC, NGC 3680, has a Gd measurement in the literature. Mitschang et al. (2012) find a $[\mathrm{Gd} / \mathrm{Fe}]=-$ $0.45 \pm 0.09$ dex, which falls significantly below the range of our $[\mathrm{Gd} / \mathrm{Fe}]$ measurements even for clusters with similar properties $\left(\sim 1.5 \mathrm{Gyr}, \mathrm{R}_{\mathrm{GC}}=8 \mathrm{kpc}\right)$. This Gd abundance relies on a single line at $4463 \AA$. Den Hartog et al. (2006) calculate atomic data for this line but do not report a solar abundance measurement, and we find that this line is too severely blended for measurement in our sample stars. There are literature measurements of Gd in metal-poor, r-process enriched stars, but they do not cover the same metallicity range as our OC and are more difficult to place within the context of this study, so we do not include them here.

To our knowledge, no measurements of open cluster Dy abundances are present in the literature. François et al. (2007) have a compilation of literature measurements suggesting that $[\mathrm{Dy} / \mathrm{Fe}]$ abundances decrease with increasing $[\mathrm{Fe} / \mathrm{H}]$ and also show less scatter, although the abundance measurements are for stars with $-4<[\mathrm{Fe} / \mathrm{H}]<-1$, well outside of the typical OC metallicity range. The $[\mathrm{Dy} / \mathrm{Fe}]$ abundances appear to be approaching solar at an $[\mathrm{Fe} / \mathrm{H}]$ of -1 .

Very little observational data for Mo is available in the literature due to the difficulty of measuring weak, singly ionized Mo lines in the optical. One cluster from our sample, NGC 7789, has a Pr abundance measurement in the literature, from Tautvaišienè et al. (2005). Again, the two abundance determinations agree within the errors. Tautvaišienè et al. (2005) measure the $5322 \AA$ line using atomic data from the VALD.

The Reddy et al. (2012, 2013, 2015) OC sample also includes Nd abundances. The Nd data in the literature is measured via equivalent widths, and as we mentioned previously $\mathrm{Nd}$ has minimal hyperfine and isotopic splitting, so we have simply corrected these based on the offset in solar abundance measurements for $[\mathrm{Nd} / \mathrm{Fe}]$, which is negligible here. Our Nd abundances match up well with the handful available in the literature for our OCs with differences on the order of $\sim 0.05$ dex which do not appear to be systematic. The Reddy et al. (2012, 2013, 2015) and Pancino et al. (2010) Nd abundances are based on a few lines with atomic data from Den Hartog et al. (2003), and Carrera \& Pancino (2011) use VALD atomic data that is very similar for the three lines measured, so this agreement is reassuring but not surprising.

\subsection{Age Trends}

In Figure 5, we plot our $[\mathrm{n}-/ \mathrm{Fe}]$ ratios vs. cluster age (circles) with error bars marking the cluster dispersions (for clusters with more than one star) or the stellar dispersions (for clusters with only one star). Also plotted are literature OC measurements from Reddy et al. (2012, 2013, 2015) and Carretta et al. (2007) (squares) and Cepheid abundances from Andrievsky et al. (2002a,b,c, 2004) and Luck et al. (2003) for Eu, Gd, and Nd and Luck \& Lambert (2011) for Pr (triangles). We have assumed a typical Cepheid age of 200 Myr, and the error bars on the Cepheid points represent the standard deviations of all Cepheid abundance measurements. The black line marks the best fit to our data, and the gray lines show the $95 \%$ confidence interval on the slope.

If r-process elements trace the remnants of massive stars (type II supernovae or neutron stars, $\mathrm{M}>8 \mathrm{M}_{\odot}$ ) and iron mainly traces lower mass supernovae (type Ia, $\mathrm{M}<8 \mathrm{M}_{\odot}$ ), we might expect that through the history of the Galaxy early clusters will form with high levels of r-process enrichment from a few massive stars but progressively higher levels of iron as longer and longer-lived low-mass stars pollute the ISM. Thus, we may see a decrease in $[\mathrm{r}-/ \mathrm{Fe}]$ for young clusters. The selected literature sample does not have the same coverage of intermediate and old OCs as ours (the single old OC at 10 Gyr is NGC 6791) but it is consistent with our data set.

Table 11 shows the linear regression fit parameters for our abundance trends with age and Galactocentric radius. The $[\mathrm{Eu} / \mathrm{Fe}]$ trend with age based on our OC data is on the edge of statistical significance $(\mathrm{p}=0.039)$ with a slope of $0.024 \pm 0.011 \mathrm{dex} \mathrm{Gyr}^{-1}$. Jacobson \& Friel (2013) find a similar [Eu/Fe] trend with age of 0.023 dex $\mathrm{Gyr}^{-1}$ but with a higher $\mathrm{p}=0.074$. We were able to reduce the dispersions in clusters for which we obtained additional spectra (N1817, N2141, N6939), and the addition of N6192 at 0.18 Gyr also makes the trend in the expanded data set more compelling. Yong et al. (2012) find an age trend in their sample [Eu/Fe] plus literature data of $-0.01 \pm 0.01$ dex $\mathrm{Gyr}^{-1}$, but the age range of their clusters is $\sim 2$ to 7 Gyr with a handful of literature clusters outside of that age range. Our trend with age is influenced by nine clusters younger than 2 Gyr which display significantly lower $[\mathrm{Eu} / \mathrm{Fe}]$ as a group than the intermediate age clusters 2 to 7 Gyr. On aver- 
Table 9. Cluster Average Abundances

\begin{tabular}{|c|c|c|c|c|c|c|c|c|c|c|c|c|c|c|}
\hline Cluster & $\begin{array}{c}\text { Avg. } \\
{[\mathrm{Fe} / \mathrm{H}]}\end{array}$ & $\sigma \mathrm{Fe}$ & $\begin{array}{c}\text { Avg. } \\
{[\mathrm{Eu} / \mathrm{Fe}]}\end{array}$ & $\sigma \mathrm{Eu}$ & $\begin{array}{c}\text { Avg. } \\
{[\mathrm{Gd} / \mathrm{Fe}]}\end{array}$ & $\sigma \mathrm{Gd}$ & $\begin{array}{c}\text { Avg. } \\
{[\mathrm{Dy} / \mathrm{Fe}]}\end{array}$ & $\sigma \mathrm{Dy}$ & $\begin{array}{c}\text { Avg. } \\
{[\mathrm{Mo} / \mathrm{Fe}]}\end{array}$ & $\sigma \mathrm{Mo}$ & $\begin{array}{c}\text { Avg. } \\
{[\mathrm{Pr} / \mathrm{Fe}]}\end{array}$ & $\sigma \operatorname{Pr}$ & $\begin{array}{c}\text { Avg. } \\
{[\mathrm{Nd} / \mathrm{Fe}]}\end{array}$ & $\sigma \mathrm{Nd}$ \\
\hline Be17 & -0.12 & 0.01 & -0.01 & 0.09 & $\ldots$ & $\ldots$ & $\ldots$ & $\ldots$ & -0.03 & 0.04 & -0.13 & 0.06 & -0.02 & 0.08 \\
\hline Be18 & -0.32 & 0.03 & 0.11 & 0.04 & 0.22 & 0.01 & 0.23 & 0.01 & 0.09 & 0.05 & 0.20 & 0.02 & 0.20 & 0.03 \\
\hline Be21 & -0.21 & 0.10 & 0.04 & 0.06 & 0.29 & 0.04 & 0.17 & 0.03 & 0.13 & 0.06 & 0.18 & 0.01 & 0.20 & 0.01 \\
\hline $\mathrm{Be} 22$ & -0.27 & 0.04 & 0.26 & 0.04 & 0.23 & 0.05 & 0.26 & 0.07 & 0.12 & 0.08 & 0.27 & 0.07 & 0.36 & 0.07 \\
\hline Be31 & -0.35 & $\ldots$ & 0.27 & 0.07 & $\ldots$ & $\ldots$ & $\ldots$ & $\ldots$ & 0.46 & 0.17 & $\ldots$ & $\ldots$ & 0.56 & 0.08 \\
\hline Be32 & -0.26 & 0.06 & 0.08 & 0.07 & 0.32 & 0.03 & 0.37 & 0.06 & 0.06 & 0.07 & 0.01 & 0.14 & 0.06 & 0.11 \\
\hline Be39 & -0.13 & 0.02 & 0.04 & 0.06 & $\ldots$ & $\ldots$ & $\ldots$ & $\ldots$ & 0.11 & 0.09 & 0.07 & 0.09 & 0.06 & 0.03 \\
\hline Cr261 & -0.06 & 0.04 & -0.07 & 0.07 & $\ldots$ & $\ldots$ & $\ldots$ & $\ldots$ & -0.05 & 0.04 & -0.30 & 0.01 & -0.10 & 0.00 \\
\hline M67 & 0.05 & 0.04 & -0.11 & 0.04 & $\ldots$ & $\ldots$ & $\ldots$ & $\ldots$ & 0.05 & 0.03 & -0.06 & 0.01 & 0.00 & 0.02 \\
\hline N188 & 0.12 & 0.04 & -0.12 & 0.07 & $\ldots$ & $\ldots$ & $\ldots$ & $\ldots$ & 0.23 & 0.10 & -0.05 & 0.04 & 0.14 & 0.05 \\
\hline N1193 & -0.17 & $\ldots$ & -0.03 & 0.09 & $\ldots$ & $\ldots$ & $\ldots$ & $\ldots$ & 0.28 & 0.08 & 0.14 & 0.10 & 0.05 & 0.07 \\
\hline N1245 & 0.02 & 0.03 & -0.12 & 0.09 & $\ldots$ & $\ldots$ & $\ldots$ & $\ldots$ & 0.12 & 0.08 & 0.07 & 0.12 & 0.08 & 0.08 \\
\hline N1817 & $-0.05^{\mathrm{a}}$ & 0.02 & -0.08 & 0.04 & 0.04 & 0.02 & -0.02 & 0.00 & 0.25 & 0.07 & 0.07 & 0.07 & 0.15 & 0.01 \\
\hline N1883 & -0.04 & 0.01 & -0.20 & 0.03 & $\ldots$ & $\ldots$ & $\ldots$ & $\ldots$ & -0.04 & 0.21 & 0.04 & 0.02 & 0.01 & 0.02 \\
\hline N2141 & $-0.09^{a}$ & 0.01 & -0.16 & 0.05 & 0.04 & 0.03 & $\ldots$ & $\ldots$ & -0.09 & 0.03 & -0.15 & 0.13 & -0.06 & 0.04 \\
\hline N2158 & -0.05 & $\ldots$ & -0.23 & 0.08 & $\ldots$ & $\ldots$ & $\ldots$ & $\ldots$ & -0.02 & 0.04 & 0.00 & 0.06 & 0.06 & 0.08 \\
\hline N2194 & -0.06 & 0.00 & -0.23 & 0.09 & $\ldots$ & $\ldots$ & $\ldots$ & $\ldots$ & 0.25 & 0.05 & 0.12 & 0.02 & 0.19 & 0.08 \\
\hline N2355 & -0.04 & 0.10 & -0.06 & 0.07 & $\ldots$ & $\ldots$ & $\ldots$ & $\ldots$ & 0.29 & 0.10 & 0.18 & 0.13 & 0.20 & 0.14 \\
\hline N6192 & 0.10 & 0.05 & -0.11 & 0.07 & $\ldots$ & $\ldots$ & $\ldots$ & $\ldots$ & 0.05 & 0.10 & -0.03 & 0.10 & 0.04 & 0.10 \\
\hline N6939 & $0.03^{\mathrm{a}}$ & 0.06 & -0.25 & 0.10 & -0.09 & 0.11 & -0.11 & 0.01 & -0.10 & 0.19 & -0.08 & 0.14 & -0.07 & 0.11 \\
\hline N7142 & 0.08 & 0.02 & -0.14 & 0.02 & $\ldots$ & $\ldots$ & $\ldots$ & $\ldots$ & 0.14 & 0.03 & -0.02 & 0.04 & 0.01 & 0.02 \\
\hline N7789 & 0.00 & 0.03 & -0.11 & 0.03 & 0.17 & 0.03 & 0.03 & 0.03 & 0.22 & 0.12 & 0.12 & 0.06 & 0.16 & 0.03 \\
\hline PWM4 & -0.18 & $\ldots$ & 0.10 & 0.01 & 0.07 & 0.05 & 0.18 & $\ldots$ & -0.06 & 0.04 & 0.12 & 0.09 & 0.14 & 0.06 \\
\hline
\end{tabular}

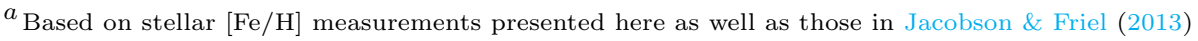

Table 10. Literature Abundance Comparisons for Clusters

\begin{tabular}{lcrrrrrrr}
\hline \hline Cluster & Source $^{\mathrm{a}}$ & $\Delta[\mathrm{Fe} / \mathrm{H}]$ & $\Delta[\mathrm{Eu} / \mathrm{Fe}]$ & $\sigma_{E u}{ }^{\mathrm{b}}$ & $\Delta[\mathrm{Pr} / \mathrm{Fe}]$ & $\sigma_{P r}{ }^{\mathrm{b}}$ & $\Delta[\mathrm{Nd} / \mathrm{Fe}]$ & $\sigma_{N d}{ }^{\mathrm{b}}$ \\
\hline $\mathrm{Be} 18$ & $\mathrm{Y} 12$ & -0.14 & 0.22 & 0.06 & $\ldots$ & $\ldots$ & $\ldots$ & $\ldots$ \\
$\mathrm{Be} 21$ & $\mathrm{Y} 12$ & -0.12 & 0.30 & 0.08 & $\ldots$ & $\ldots$ & $\ldots$ & $\ldots$ \\
$\mathrm{Be} 22$ & $\mathrm{Y} 12$ & -0.19 & 0.03 & 0.10 & $\ldots$ & $\ldots$ & $\ldots$ & $\ldots$ \\
$\mathrm{B} 31$ & $\mathrm{Y} 05$ & -0.24 & 0.32 & $\ldots$ & $\ldots$ & $\ldots$ & $\ldots$ & $\ldots$ \\
$\mathrm{B} 32$ & $\mathrm{Y} 12$ & -0.14 & 0.26 & 0.11 & $\ldots$ & $\ldots$ & $\ldots$ & $\ldots$ \\
Be32 & $\mathrm{CP} 11$ & -0.06 & $\ldots$ & $\ldots$ & $\ldots$ & $\ldots$ & -0.08 & 0.06 \\
M67 & PCRG10 & -0.02 & $\ldots$ & $\ldots$ & $\ldots$ & $\ldots$ & 0.03 & 0.05 \\
M67 & T00 & -0.09 & 0.12 & 0.07 & $\ldots$ & $\ldots$ & $\ldots$ & $\ldots$ \\
M67 & Y05 & -0.05 & 0.20 & 0.02 & $\ldots$ & $\ldots$ & $\ldots$ & $\ldots$ \\
M67 & RGL & -0.11 & -0.11 & $\ldots$ & $\ldots$ & $\ldots$ & 0.01 & 0.04 \\
N1817 & RGL & -0.06 & -0.09 & $\ldots$ & $\ldots$ & $\ldots$ & -0.02 & 0.04 \\
N2141 & Y05 & -0.11 & 0.36 & $\ldots$ & $\ldots$ & $\ldots$ & $\ldots$ & $\ldots$ \\
N7789 & PCRG10 & 0.02 & $\ldots$ & $\ldots$ & $\ldots$ & $\ldots$ & -0.04 & 0.30 \\
N7789 & T05 & -0.04 & 0.13 & 0.12 & -0.04 & 0.05 & $\ldots$ & $\ldots$ \\
PWM4 & Y12 & -0.18 & 0.05 & $\ldots$ & $\ldots$ & $\ldots$ & $\ldots$ & $\ldots$ \\
\hline
\end{tabular}

${ }^{a}$ References for literature comparisons: CP11 = Carrera \& Pancino (2011); PCRG10 = Pancino et al. (2010); RGL = Reddy et al. (2012, 2013); T00 = Tautvaišienè et al. (2000); T05 = Tautvaišienè et al. (2005); Y05 = Yong et al. (2005)

$b$ These columns are the errors on cluster measurements given in the literature sources. 
age, we find the younger clusters have $[\mathrm{Eu} / \mathrm{Fe}] \sim 0.2$ dex lower than the intermediate ages. Thus, the difference in trends could be a cluster selection effect. It is also possible that the observed element-to-iron ratios have a non-linear relationship with age, as the decrease in $[\mathrm{Eu} / \mathrm{Fe}]$ for clusters younger than 2 Gyr suggests there might be a break there, but the scatter in our abundance measurements is too large to allow us to evaluate goodness of fit for different models.

We also recalculated the best fit to the $[\mathrm{Eu} / \mathrm{Fe}]$ abundances with age using only stellar measurements of the $6645 \AA$ line. As mentioned previously, most literature studies rely only on the $6645 \AA$ line when measuring Eu abundances in metal-rich stars. We find that this makes $\sim 0.001$ dex $\mathrm{Gyr}^{-1}$ difference to the slope and the error on the slope. The y-intercept changes by 0.01 dex. We conclude that including other, more blended lines isn't distorting our determined Eu trend with age.

The Cepheid $[\mathrm{Gd} / \mathrm{Fe}]$ measurements are similar to values we find for our young open clusters. These data cannot be well placed in the context of the enrichment of the Galaxy with time like the OC data, but it is heartening that they reproduce the same abundance range as our data for this relatively understudied element. Unfortunately, due to the limited number of clusters for which we could measure Gd, the trend is not statistically significant $(\mathrm{p}=0.240)$ although it is similar in strength to $\mathrm{Eu}$, with a slope of $0.026 \pm 0.021$. We must be cautious about interpreting such limited data, but the fact that the cluster abundances for $\mathrm{Eu}$ and Gd track each other is interesting.

Due to the wavelength and signal-to-noise restrictions of our data, only eight clusters have Dy measurements presented here. The $[\mathrm{Dy} / \mathrm{Fe}]$ data show a stronger and more significant increase with age than Eu or Gd, with a slope of $0.054 \pm 0.017$ and $p=0.018$, although it should be noted that the $[\mathrm{Dy} / \mathrm{H}]$ abundances are all within 0.1 dex of solar. Once again, the sparse data requires careful interpretation, but the Dy and Gd abundances track each other very well, as we would expect of the r-process elements of similar weight. If the r-process does indeed have multiple sites, those elements and isotopes with similar neutron numbers should still have roughly the same yields.

Because $32 \%$ of solar Mo is produced by the r-process (see Section 4.4), it is not considered a majority sprocess element but would be expected to display abundance patterns somewhere between the pure r- and sprocess elements. We find that $[\mathrm{Mo} / \mathrm{Fe}]$ shows a statistically insignificant increase with decreasing cluster age, the best linear fit to the data having a slope consistent with zero $\left(-0.012 \pm 0.012 \mathrm{dex} \mathrm{Gyr}^{-1}\right)$. The trend is also influenced by the oldest cluster, Be 17, which has abundances that fall slightly below the best fit line for all elements measurable in the KPNO data. We must not place too much weight on the effects of this single cluster; when it is excluded, there is no visual suggestion of a trend with age.

$\mathrm{Pr}$ and $\mathrm{Nd}$ are about half-and-half $\mathrm{r}-$ and s- process, so again we would expect their trends with age to fall between the pure $\mathrm{r}$ - and s- process elements similar to Mo. Both exhibit statistically insignificant trends $(\mathrm{p}=$ 0.321 and 0.537 ) and their slopes are consistent with zero within the errors $(-0.011 \pm 0.011$ and $-0.008 \pm 0.012 \mathrm{dex}$ $\mathrm{Gyr}^{-1}$, respectively). Be 17 also falls below the trend line for these elements, and when it is removed these element-to-iron ratios appear to have no relationship with cluster age. The Nd literature OC and Cepheid data match the abundances of our youngest clusters well. The Pr Cepheid data falls a bit below the typical abundances for young clusters, but the dispersion among the individual Cepheids for both $\mathrm{Pr}$ and $\mathrm{Nd}$ is consistent with the dispersion among the OCs.

If we consider the Jacobson \& Friel (2013) s-process trends with age for $\mathrm{Zr}$, La, and $\mathrm{Ba}(-0.011,-0.015$, and -0.036 dex $\mathrm{Gyr}^{-1}$ respectively), then it seems the mixed $\mathrm{r}$ - and s- process elements do fall between the trends on the pure $\mathrm{r}-$ and s- elements, although the uncertainties on the slopes of the mixed elements prevent any strong conclusions. We find the dispersion about the trend lines with age for the neutron-capture elements is $\sim 0.10-0.15$ dex, which is larger than many of the cluster dispersions but similar to the size of the errors expected from atmospheric parameter uncertainties. It is also about the same size as the abundance change in the trend line over the $10 \mathrm{Gyr}$ age range of our clusters, offering further evidence that the mixed elements have no trends with age.

It is interesting to note that our Be 31 abundances (based on a single star) are the highest of any OC for the three elements that had lines in the limited wavelength region for this stellar spectrum. The Be 31 [Eu/Fe] abundance is only slightly higher than Be 22, but its $[\mathrm{Mo} / \mathrm{Fe}]$ and $[\mathrm{Nd} / \mathrm{Fe}]$ abundances are $\sim 0.2$ dex higher than any other cluster in our sample. Because it is in the intermediate age range (2.6 Gyr) it does not have a large effect on element trends with age, but its location in the outer disk does strongly influence determined trends with Galactocentric radius which we discuss in the next section.

\subsection{Galactocentric Radius Effects 7.3.1. Iron}

In Figure 6 we plot $[\mathrm{Fe} / \mathrm{H}]$ abundances for our sample clusters, selected literature OC data, and Andrievsky et al. (2002a,b,c, 2004) and Luck et al. (2003) Cepheid data against Galactocentric radius, using the same symbols as Figure 5. Our clusters have $\mathrm{R}_{\mathrm{GC}} \sim 7$ to $16 \mathrm{kpc}$, with the furthest cluster being Be 31. Several studies 


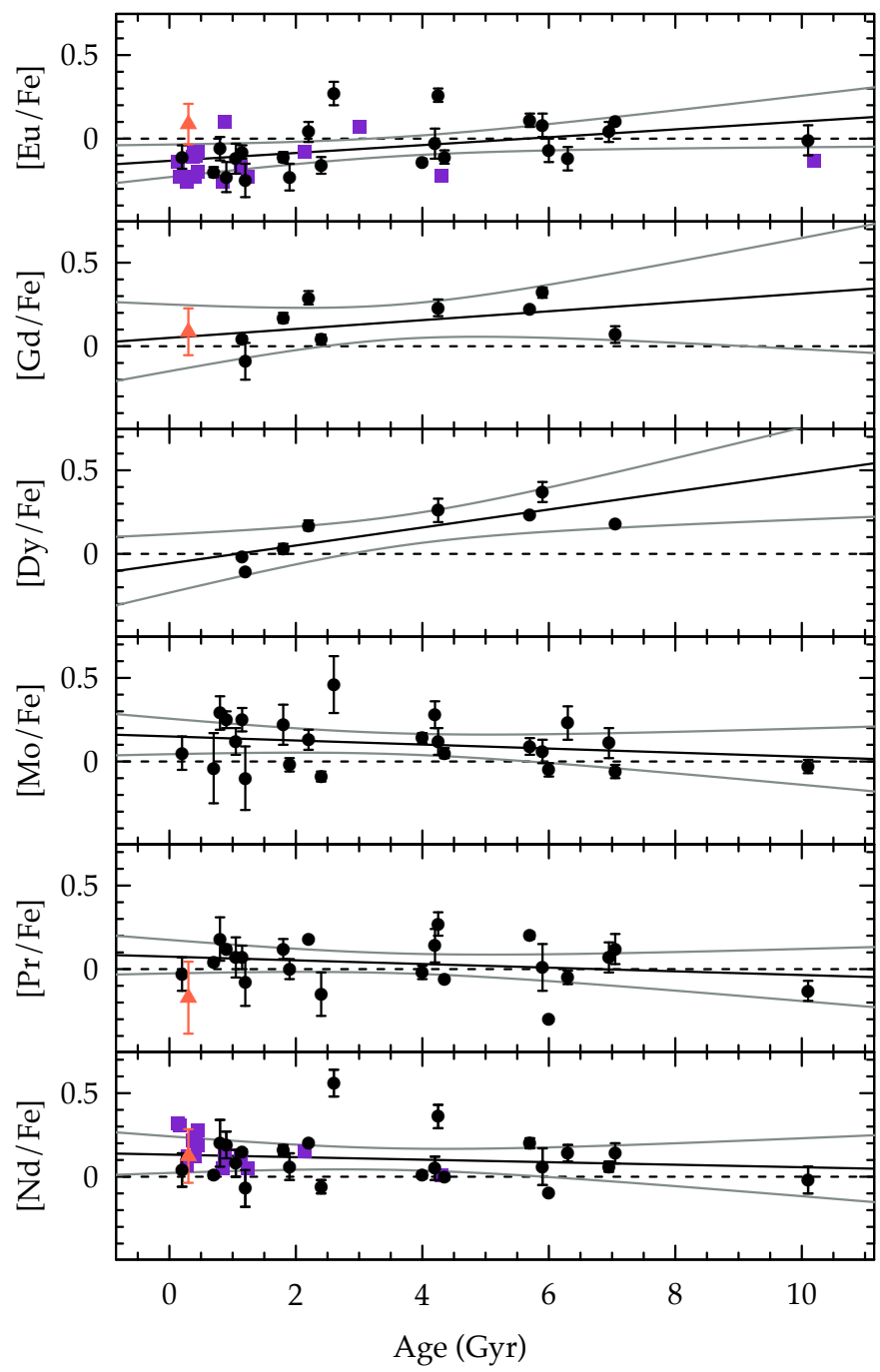

Figure 5. Abundance trends with age for OCs from this paper (black with error bars indicating cluster dispersions), for literature OCs from Reddy et al. (2012, 2013, 2015) and Carretta et al. (2007) adjusted to our abundance scale (squares), and Cepheids from Andrievsky et al. (2002a,b,c, 2004) and Luck et al. (2003) (triangles, Eu, Gd and Nd) and Luck \& Lambert (2011) (triangles, Pr). The solid black line marks the best fit trend, and gray lines mark the $95 \%$ confidence interval on the slope.

of the Galactic Fe gradient have noted a change in the gradient at $\sim 10$ to $13 \mathrm{kpc}$ (Twarog et al. 1997; Friel et al. 2010; Carrera \& Pancino 2011; Yong et al. 2012). Our data do not extend far enough into the outer disk to assess whether our measured $[\mathrm{Fe} / \mathrm{H}]$ distribution is indeed best fit by two linear regressions instead of one, so in Figure 6 we mark a single linear regression to our OC data set as a solid line, and the $[\mathrm{Fe} / \mathrm{H}]$ fit with $\mathrm{R}_{\mathrm{GC}}$ with a break at $13 \mathrm{kpc}$ from Yong et al. (2012) as dashed lines. Our cluster sample also does not include any extremely high metallicity OCs, but Carretta et al. (2007) have two inner disk clusters with $[\mathrm{Fe} / \mathrm{H}]>0.4 \mathrm{dex}, \mathrm{NGC}$ 6253 and NGC 6791. These are old open clusters with ages of $\sim 3$ and $10 \mathrm{Gyr}$ and abundances based on 4 member stars each; NGC 6791 is the only open cluster in the sample of Salaris et al. (2004) found to be older than
Berkeley 17.

Supplementing our $[\mathrm{Fe} / \mathrm{H}]$ data with Cepheid data over the same distance range, we do see a suggestion of a leveling out around $13 \mathrm{kpc}$. The Cepheid [Fe/H] abundances also decrease more sharply with $\mathrm{R}_{\mathrm{GC}}$ than our $\mathrm{OC}$ abundances. The slope of the $[\mathrm{Fe} / \mathrm{H}]$ gradient found for $\mathrm{R}_{\mathrm{GC}}<13 \mathrm{kpc}$ in Yong et al. (2012) is -0.09 dex $\mathrm{Gyr}^{-1}$; from our OC data alone, we find $-0.047 \pm$ 0.007 dex $\mathrm{Gyr}^{-1}$, but the Andrievsky et al. (2002a,b,c, 2004) and Luck et al. (2003) Cepheid data gives 0.06 dex $\mathrm{Gyr}^{-1}$. Our clusters fall roughly in line with the Cepheid $[\mathrm{Fe} / \mathrm{H}]$ at different radii, so the difference in slopes may be due to the relative sparseness of inner disk OCs in our sample.

\subsection{2. r-process elements}


Table 11. Cluster Linear Regression Parameters

\begin{tabular}{lrrrrrrrr}
\hline \hline \multicolumn{1}{c}{ Variables } & Slope & $\sigma_{\text {slope }}$ & Intercept & $\sigma_{\text {int. }}$ & $\mathrm{R}$ & $\mathrm{P}$-value & $\mathrm{N}$ & $\sigma_{\text {resid. }}$ \\
\hline$[\mathrm{Mo} / \mathrm{Fe}]$ vs. Age & -0.012 & 0.012 & 0.150 & 0.051 & -0.222 & 0.310 & 23 & 0.141 \\
{$[\mathrm{Pr} / \mathrm{Fe}]$ vs. Age } & -0.011 & 0.011 & 0.074 & 0.048 & -0.222 & 0.321 & 22 & 0.130 \\
{$[\mathrm{Nd} / \mathrm{Fe}]$ vs. Age } & -0.008 & 0.012 & 0.132 & 0.053 & -0.136 & 0.537 & 23 & 0.145 \\
{$[\mathrm{Eu} / \mathrm{Fe}]$ vs. Age } & 0.024 & 0.011 & -0.133 & 0.047 & 0.434 & 0.039 & 23 & 0.129 \\
{$[\mathrm{Gd} / \mathrm{Fe}]$ vs. Age } & 0.026 & 0.021 & 0.050 & 0.084 & 0.437 & 0.240 & 9 & 0.122 \\
{$[\mathrm{Dy} / \mathrm{Fe}]$ vs. Age } & 0.054 & 0.017 & -0.058 & 0.071 & 0.795 & 0.018 & 8 & 0.097 \\
{$[\mathrm{Fe} / \mathrm{H}]$ vs. $R_{\mathrm{GC}}$} & -0.047 & 0.007 & 0.439 & 0.078 & -0.831 & 0.000 & 23 & 0.074 \\
{$[\mathrm{Mo} / \mathrm{Fe}]$ vs. $R_{\mathrm{GC}}$} & 0.015 & 0.013 & -0.065 & 0.149 & 0.250 & 0.250 & 23 & 0.140 \\
{$[\mathrm{Pr} / \mathrm{Fe}]$ vs. $\mathrm{R}_{\mathrm{GC}}$} & 0.039 & 0.011 & -0.393 & 0.122 & 0.624 & 0.002 & 22 & 0.104 \\
{$[\mathrm{Nd} / \mathrm{Fe}]$ vs. $R_{\mathrm{GC}}$} & 0.039 & 0.011 & -0.331 & 0.122 & 0.623 & 0.001 & 23 & 0.115 \\
{$[\mathrm{Eu} / \mathrm{Fe}]$ vs. $\mathrm{R}_{\mathrm{GC}}$} & 0.039 & 0.010 & -0.492 & 0.116 & 0.648 & 0.001 & 23 & 0.109 \\
{$[\mathrm{Gd} / \mathrm{Fe}]$ vs. $\mathrm{R}_{\mathrm{GC}}$} & 0.038 & 0.019 & -0.315 & 0.232 & 0.603 & 0.086 & 9 & 0.109 \\
{$[\mathrm{Dy} / \mathrm{Fe}]$ vs. $\mathrm{R}_{\mathrm{GC}}$} & 0.050 & 0.020 & -0.457 & 0.241 & 0.716 & 0.046 & 8 & 0.111 \\
\hline
\end{tabular}

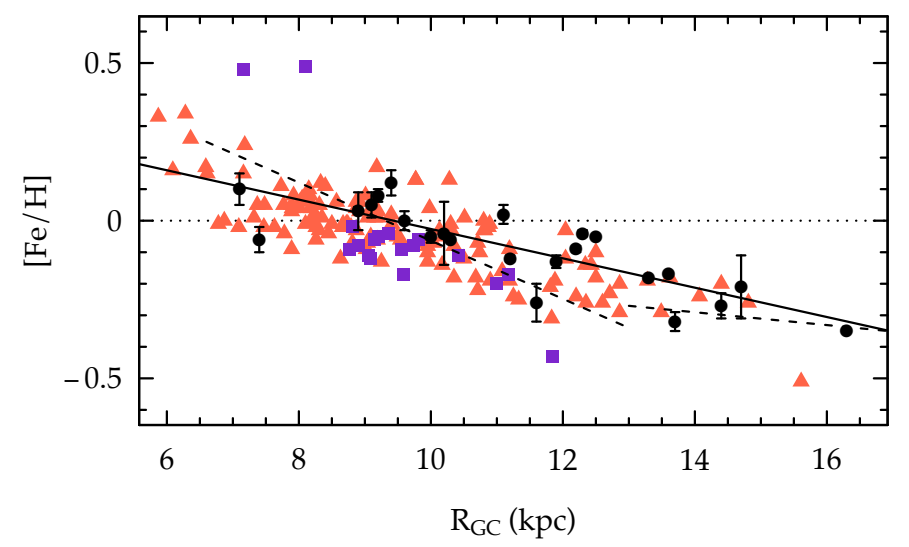

Figure 6. Our cluster $[\mathrm{Fe} / \mathrm{H}]$ abundances with Galactocentric radius; the solid line represents a linear fit to the data (see text) and the dashed lines are an iron gradient fit with a break at $13 \mathrm{kpc}$ from Yong et al. (2012). Symbols are the same as Figure 5 except here triangles represent individual Cepheid measurements.

In Figure 7, we plot our [n-capture/Fe] ratios vs. Galactocentric radius (circles) with error bars marking the cluster dispersions (for clusters with more than one star) or the stellar dispersions (for clusters with only one star), the black lines marking the best fit to our data, and the gray lines showing the $95 \%$ confidence interval on the slopes.

We find a $[\mathrm{Eu} / \mathrm{Fe}]$ with $\mathrm{R}_{\mathrm{GC}}$ trend of $0.039 \pm 0.010 \mathrm{dex}$ $\mathrm{kpc}^{-1}$ and p-value of 0.001 , which seems to be driven by clusters beyond $13 \mathrm{kpc}$. Jacobson \& Friel (2013) find a trend with $\mathrm{R}_{\mathrm{GC}}$ of $0.047 \mathrm{dex} \mathrm{kpc}^{-1}$ with a p-value of 0.017. Yong et al. (2012) fit two regressions to $[\mathrm{Eu} / \mathrm{Fe}]$ with $\mathrm{R}_{\mathrm{GC}}$ for the apparent break in $[\mathrm{Fe} / \mathrm{H}]$ at $13 \mathrm{kpc}$, but since our clusters do not extend as far into the outer disk we do not calculate the separate regressions; the slope of their regression for $\mathrm{R}_{\mathrm{GC}}<13 \mathrm{kpc}$ is $0.07 \pm 0.01 \mathrm{dex}$

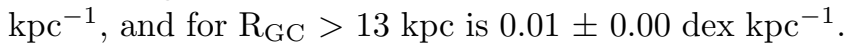
Their sample covers mainly intermediate-distance clus- ters with $11<\mathrm{R}_{\mathrm{GC}}<16 \mathrm{kpc}$, with one inner and outer cluster from their data set and four inner disk clusters drawn from the literature.

Attempting to correct for all systematic differences between their sample and the literature was not the goal of the work, so there may be some remaining differences causing a disparity between their intermediate $\mathrm{R}_{\mathrm{GC}}$ clusters and inner disk clusters from the literature. As discussed previously, due to differences in atomic data and line measurement techniques, we have seen variations in $[\mathrm{Eu} / \mathrm{Fe}]$ measurements up to 0.3 dex between sources. If we consider a break in the $[\mathrm{Eu} / \mathrm{Fe}]$ gradient at $13 \mathrm{kpc}$, we find an insignificant inner gradient of $0.003 \pm 0.014$ dex $\mathrm{kpc}^{-1}$ and a strong outer gradient for the handful of clusters we have of $0.069 \pm 0.042 \mathrm{dex} \mathrm{kpc}^{-1}$. However, considering only the clusters with $[\mathrm{Eu} / \mathrm{Fe}]$ measurements by Yong et al. $(2005,2012)$ over the Galactocentric radius range of our data, we find a best fit slope of 0.031 
$\pm 0.023 \mathrm{dex} \mathrm{kpc}^{-1}$, which is consistent with our single regression slope.

We have again calculated the $[\mathrm{Eu} / \mathrm{Fe}]$ trend with $\mathrm{R}_{\mathrm{GC}}$ based only on the $6645 \AA$ line. We find no significant difference from the trend derived from all four lines; $[\mathrm{Eu} / \mathrm{Fe}]_{6645}=0.035 \pm 0.010 \times \mathrm{R}_{\mathrm{GC}}-0.430 \pm 0.114$.

Our sample $[\mathrm{Gd} / \mathrm{Fe}]$ trend with $\mathrm{R}_{\mathrm{GC}}$ matches the $[\mathrm{Eu} / \mathrm{Fe}]$ trend, though with a lower statistical significance, with a slope of $0.038 \pm 0.019 \mathrm{dex} \mathrm{Gyr}^{-1}$ and pvalue of 0.086 . Our $[\mathrm{Dy} / \mathrm{Fe}]$ OC trend is stronger than $\mathrm{Gd}$ or $\mathrm{Eu}$, with a slope of $0.050 \pm 0.020$ dex $\mathrm{Gyr}^{-1}, \mathrm{p}$ $=0.046$.

Figure 8 plots our data over literature data with $\mathrm{R}_{\mathrm{GC}}$; sources are represented with the same symbols as Figure 5, but with triangles now representing individual Cepheid values. The literature OC abundances for $[\mathrm{Eu} / \mathrm{Fe}]$ with $\mathrm{R}_{\mathrm{GC}}$ match our determined abundances well.

The Cepheid $[\mathrm{Eu} / \mathrm{Fe}]$ data trend with $\mathrm{R}_{\mathrm{GC}}$ has a slope of $0.035 \pm 0.005 \mathrm{dex} \mathrm{kpc}^{-1}$ which is similar to our calculated value although there does appear to be some systematic offset between the two data sets. Because the Cepheid abundances were measured using only equivalent widths, this may create some systematic differences for the elements we have synthesized using hyperfine or isotopic structure.

The Cepheid $[\mathrm{Gd} / \mathrm{Fe}]$ measurements do not cover the full Galactocentric radius range of our cluster sample, but they are similar to values we find for our open clusters over the range of the Cepheid data $(\sim 7-11 \mathrm{kpc})$. The Cepheid $[\mathrm{Gd} / \mathrm{Fe}]$ trend with $\mathrm{R}_{\mathrm{GC}}$ matches our OC trend well with a slope of $0.041 \pm 0.010 \mathrm{dex} \mathrm{Gyr}^{-1}$ and p-value of $\sim 0.0001$.

\subsubsection{Mixed $r$ - and $s$ - elements}

$[\mathrm{Mo} / \mathrm{Fe}]$ has a statistically insignificant trend with Galactocentric radius, with a slope of $0.014 \pm 0.013 \mathrm{dex}$ $\mathrm{kpc}^{-1}(\mathrm{p}=0.306)$. There is little comparison data available for this element and the limitations on the determination of the trend with $\mathrm{R}_{\mathrm{GC}}$ means there is little we can say about its behavior.

$[\mathrm{Pr} / \mathrm{Fe}]$ and $[\mathrm{Nd} / \mathrm{Fe}]$ display much more significant trends with Galactocentric radius of $0.037 \pm 0.011 \mathrm{dex}$ $\mathrm{kpc}^{-1}$ with $\mathrm{p}$-values of 0.002 and 0.003 respectively. It is reassuring that trends for these elements are similar; $\operatorname{Pr}$ has no measurement for Be 31, but the fact that the results are still similar means that this cluster may not have a serious impact on the overall results. Praseodymium also has some Cepheid data available in the literature from Luck \& Lambert (2011), which displays a moderate $\mathrm{R}_{\mathrm{GC}}$ gradient similar to our data (0.04 dex $\mathrm{kpc}^{-1}$ ) but does not match our abundance scale. Again, the discrepancy in the scale of the data is probably due to our use of hyperfine structure in synthesis.
The literature OC and Cepheid data for Nd both match our data well; $[\mathrm{Nd} / \mathrm{Fe}]$ shows a suggestion of an upturn at $13 \mathrm{kpc}$ that is mirrored in the Cepheid data.

The cluster averages of the mixed r- and s- elements, $\mathrm{Mo}, \mathrm{Pr}$, and Nd, display significant scatter around the linear regression that cannot be explained by the dispersions of individual clusters. It is true that the standard deviations of individual stellar abundance measurements are an underestimation of the true cluster errors, particularly for clusters with two or three stars (error bars on clusters for which we have only one star are the stellar abundance dispersions), but there are still some clusters that fall far outside the $95 \%$ confidence interval on the slope (gray lines on Figs. 5 and 7 ). Though the scatter about the trends with age mostly seems random, there is some visible structure in the relationship of cluster abundances with galactocentric radius.

For Figure 7, we have assumed a simple linear relationship between $[\mathrm{X} / \mathrm{Fe}]$ and $\mathrm{R}_{\mathrm{GC}}$, but this probably does not accurately reflect the process of Galactic enrichment. Both possible r-process events (NS-NS mergers and type II supernovae) occur rarely and even for young stars the local ISM may not have been enriched by many such events; Kalogera et al. (2004) estimate a current Galactic neutron star merger rate of $180 \mathrm{Myr}^{-1}$. Diehl et al. (2006) estimate a Galactic CCSN rate of $19000 \mathrm{Myr}^{-1}$, though a recent chemical evolution model by Wehmeyer et al. (2015) estimates that only $0.1 \%$ of these are magnetorotationally driven 'jet' supernovae which would be capable of producing and ejecting significant amounts of r-process material. Thus, we may expect local inhomogeneities in the ISM.

The mixed elements in particular seem to have some structure with Galactocentric radius: there is a slight enhancement of about 0.2 dex from the linear regression around $10 \mathrm{kpc}$ from the Galactic center, a decrease of about the same amount at $12 \mathrm{kpc}$, and then another increase out to large radii. The increase at around $15 \mathrm{kpc}$ is based on a single cluster for which we only have one star (Be 31), which has no Pr abundance determined because of wavelength restrictions (see Section 4.5). The increase and decrease at 10 and $12 \mathrm{kpc}$, however, involve several clusters varying from a linear relationship. The available Cepheid data show a dispersion of $\sim 0.15$ dex within bins of $1 \mathrm{kpc}$ in Galactocentric radius; if we use this as a marker of intrinsic abundance scatter in the ISM at any given radius, Be 31 is the only cluster that really stands out. The other clusters have residuals of up to $0.2 \mathrm{dex}$, but these variations might be explained by the local abundance variations in the ISM or errors due to atmospheric parameters and continuum setting. Further data for s-process elements may help evaluate the significance of these nonlinearities.

In Figure 9, we show $[\mathrm{X} / \mathrm{Fe}]$ vs. Galactocentric ra- 


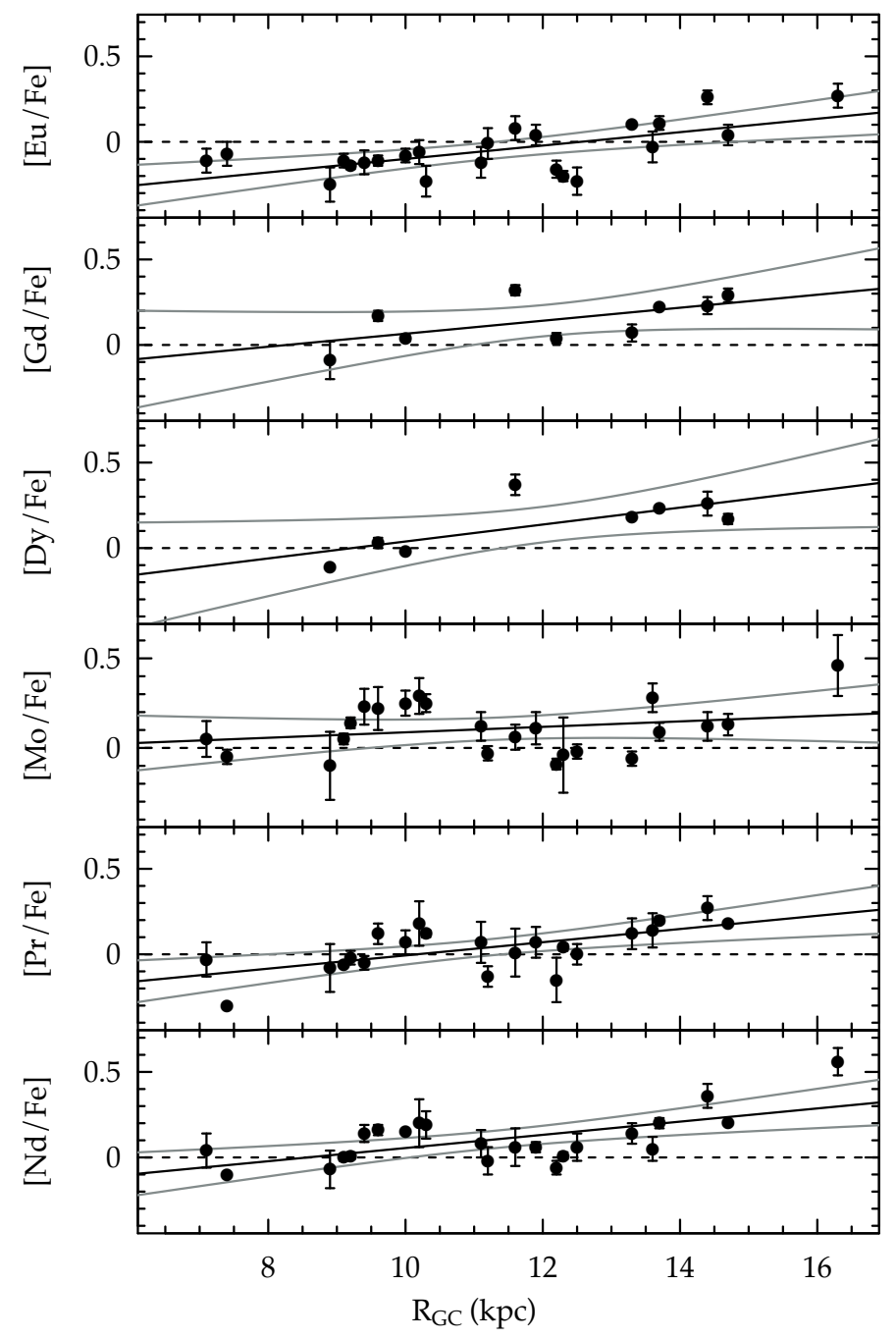

Figure 7. Abundance trends with Galactocentric radius; symbols and lines the same as in Fig. 5.

dius for all elements, with clusters color-coded by age. For elements with the full set of cluster abundances, there seems to be no trend with Galactocentric radius in the youngest clusters (age $<2$ Gyr, circles), though the intermediate age (2 - 5 Gyr, triangles) and old ( $>5$ Gyr, squares) cluster abundances increase with Galactocentric radius for $[\mathrm{Eu} / \mathrm{Fe}],[\mathrm{Pr} / \mathrm{Fe}]$, and $[\mathrm{Nd} / \mathrm{Fe}]$. More specifically, when fitting a linear regression to each age group individually, the youngest clusters have a slope consistent with zero for all elements, the intermediate age clusters have positive slopes from 0.04 (Mo) to 0.06 $(\mathrm{Nd}) \pm 0.02 \mathrm{dex} \mathrm{kpc}^{-1}$, and the oldest clusters have positive slopes for $\mathrm{Eu}(0.035 \pm 0.009), \operatorname{Pr}(0.070 \pm 0.013)$, and Nd $(0.034 \pm 0.015)$.

Because the youngest clusters mostly cover the inner disk $\left(\mathrm{R}_{\mathrm{GC}} \leq 12 \mathrm{kpc}\right)$ it is difficult to determine whether the differences in gradients for the age groups are due to mixing (or some other long-timescale effect), or whether the outer disk has a distinct star formation history from the inner disk (i.e. whether the younger clusters would show increased neutron-capture abundances if data were available at large radii). Bird et al. (2012) use simulations of perturbed and unperturbed Galactic disks to estimate a typical radial migration of $\sim 1 \mathrm{kpc}$ for disk stars, with $80 \%$ moving less than $2 \mathrm{kpc}$ in $\mathrm{R}_{\mathrm{GC}}$. They note that the perturbed disks experience the largest stellar migrations preferentially at large radii, where the disk becomes unstable; a significant fraction of outer disk stars with initial radii $>15 \mathrm{kpc}(\sim 40 \%)$ migrate more than $3 \mathrm{kpc}$. It is possible that mixing due to radial migration would require long timescales on the order of Gyr to reach equilibrium.

Wu et al. (2009) calculate orbits for a large sample of OCs, twelve of which are also in our sample (we do not include Be 31 orbital calculations because of issues with its available proper motion data; see Vande Putte et al. 2010). A few of these are highly eccentric; in particular, they find that Be 21 has an eccentricity of 


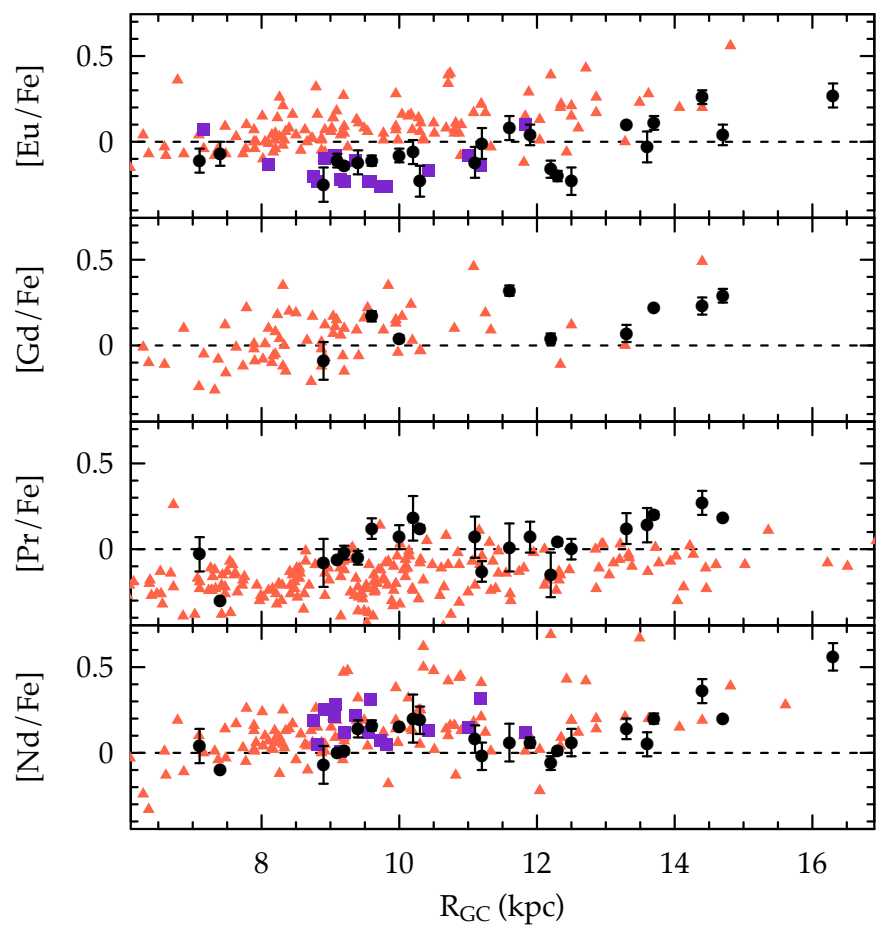

Figure 8. Abundances and literature data with Galactocentric radius; symbols and lines the same as in Fig. 5, except that triangles now represent individual Cepheid measurements.

0.47 , and calculate that it varies over $8 \mathrm{kpc}$ in its orbit from apogalacticon to perigalacticon. The median difference between $R_{\text {apo }}$ and $R_{\text {per }}$ for the dozen clusters in common is $\sim 3 \mathrm{kpc}$. This variation may also contribute significantly to the apparent 'mixing' moderating the radial gradients presented here.

There is not enough data for Gd and Dy to examine Galactocentric radius trends in the three age groups, but it is worth noting that the youngest clusters, which all fall in the 8-10 kpc range in this cluster subset, appear to be driving the overall relationship with $R_{\mathrm{GC}}$.

\subsection{Element-to-Element Ratios}

One check on the quality of our abundance measurements is the degree to which cluster abundances for elements in similar groups scale with each other. Since our group of r-process elements were presumably formed via the same nucleosynthetic mechanism, we would expect that stars showing an enhancement in one r-process element would also be enhanced in other r-process elements, and the same for mixed elements with approximately the same level of $\mathrm{r}-$ and $\mathrm{s}-$ contributions. By the same reasoning, if systematic biases are not responsible for variations in abundance measurements from star to star, we would expect to see no correlation between r-process and s-process element abundances which are produced in different astrophysical sites.

Figure 10 shows stellar r-process element abundances plotted against each other and $[\mathrm{Fe} / \mathrm{H}]$ (dotted line is a
1:1 correspondence for $\mathrm{r}-\mathrm{vs} \mathrm{r}-\mathrm{plots}$ and indicates solar ratios for $\mathrm{r}-$ vs. Fe plots). [Eu/Fe] points are plotted over disk field giant data from Mishenina et al. (2007). Though $[\mathrm{Dy} / \mathrm{Fe}]$ and $[\mathrm{Gd} / \mathrm{Fe}]$ track each other well, there appears to be an offset between these two and our $\mathrm{Eu}$ (top left and right panels) of $\sim 0.15-0.20$ dex. However, in Figs. 5 and 8 we see that the Cepheid Eu and Gd abundances have similar abundances relative to solar. If these r-process elements are formed in the same environment/location, we have no reason to expect that they would give different abundances relative to solar; we may have scaling issues due to our choice of atomic data, since the $6645 \AA$ line which most literature measurements are based on has significant hyperfine and isotopic structure, and different linelists based on many features may not give results that can be scaled simply to solar values, particularly for giant stars.

The Mishenina et al. (2007) study of 171 cool disk giants gives $[\mathrm{Eu} / \mathrm{Fe}]$ abundances systematically 0.1 to 0.2 dex higher than our values for the same $[\mathrm{Fe} / \mathrm{H}]$. Model calculations of thin disk $[\mathrm{Eu} / \mathrm{Fe}]$ from Serminato et al. (2009) also agree well with the Mishenina et al. (2007) data for all but perhaps the highest $[\mathrm{Fe} / \mathrm{H}]$ stars. $[\mathrm{Eu} / \mathrm{Fe}]$ models from Cescutti et al. (2006) predict a $[\mathrm{Eu} / \mathrm{Fe}]$ of about +0.2 at $[\mathrm{Fe} / \mathrm{H}]$ of -0.4 dex, $\sim+0.05$ at solar $[\mathrm{Fe} / \mathrm{H}]$, and -0.15 at $[\mathrm{Fe} / \mathrm{H}]$ of +0.2 dex. In particular, our stellar $[\mathrm{Eu} / \mathrm{Fe}]$ abundances at solar $[\mathrm{Fe} / \mathrm{H}]$ that fall below about -0.25 dex are not matched in the literature. Some of these stellar $[\mathrm{Eu} / \mathrm{Fe}]$ abundances are 


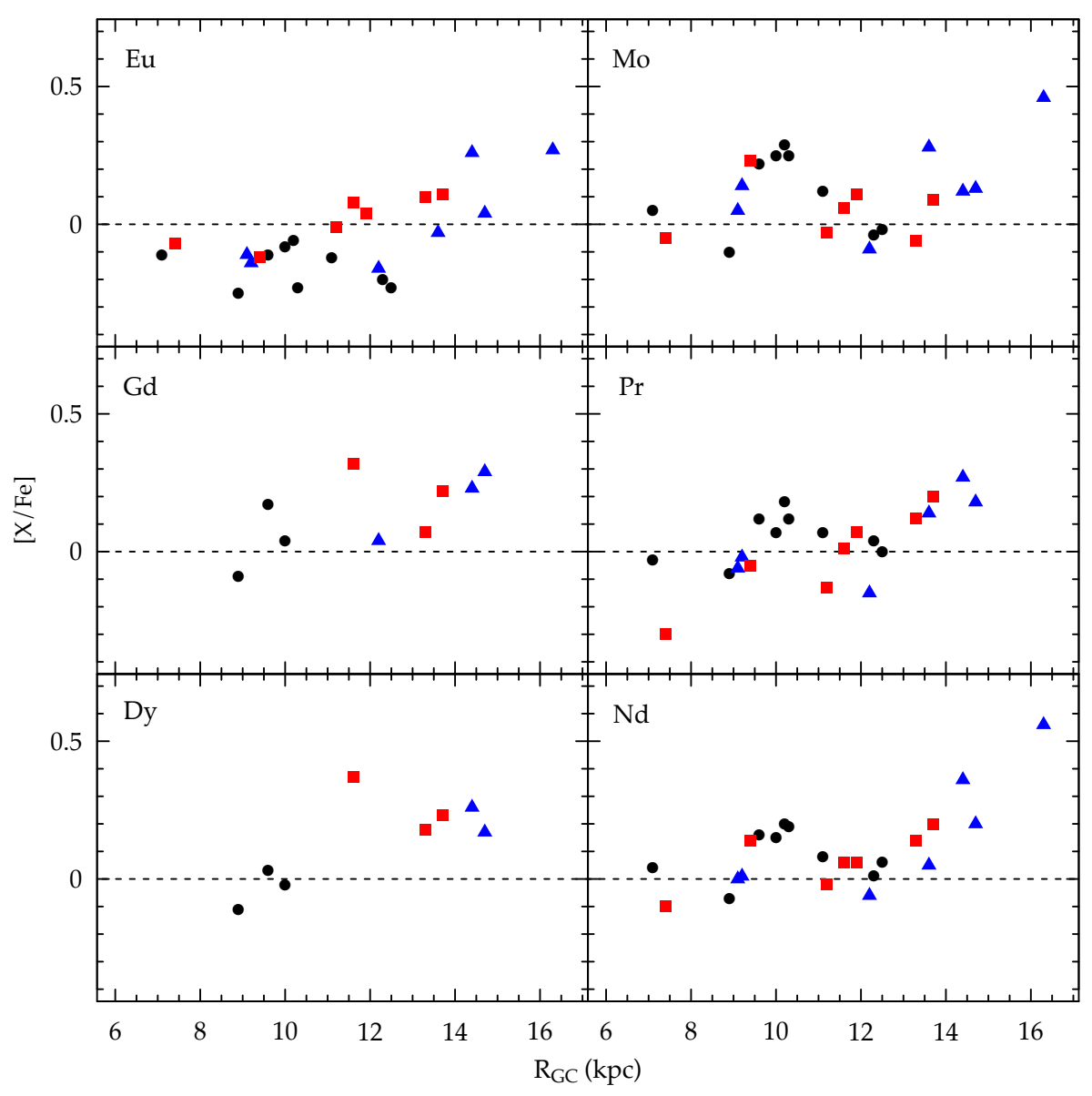

Figure 9. Same data as Fig. 7 but without error bars and separated by ages. Circles are clusters younger than 2 Gyr, triangles are $2<$ age $<5$ Gyr, and squares are older than 5 Gyr.

low compared to other members of the cluster; NGC 2158, NGC 2194, and NGC 6939 are the only clusters to have an average $[\mathrm{Eu} / \mathrm{Fe}]$ below -0.2 dex, and their internal dispersions are large enough to be consistent with the lower edge of the disk star abundances.

Interestingly, Wehmeyer et al. (2015) use a chemical model with non-instantaneous recycling and mixing to locate the r-process site by modeling the scatter in $[\mathrm{Eu} / \mathrm{Fe}]$ with time for the history of the Galaxy. The modeling is focused on the early history of the Galaxy, as the authors note that a mixture of NS-NS mergers and jet supernovae ejecta would be required to reproduce the observed scatter in $[\mathrm{Eu} / \mathrm{Fe}]$ at early times. However, they also predict a scatter in $[\mathrm{Eu} / \mathrm{Fe}]$ at solar metallicities $(-0.3<[\mathrm{Fe} / \mathrm{H}]<0.2$ dex $)$ of $\sim 0.4$ to 0.5 dex. Some of the assumptions used to model the local mixing may not hold at later times in the evolution of the Galaxy, but the close match between the predictions and our observed scatter is intriguing.

Figure 11 shows mixed element abundances plotted against each other and $[\mathrm{Fe} / \mathrm{H}] . \mathrm{Nd}$ and $\mathrm{Pr}$ correlate strongly with each other, but not as strongly with Mo. None of the three elements correlate strongly with
$[\mathrm{Fe} / \mathrm{H}]$. The correlation of $\mathrm{Pr}$ and $\mathrm{Nd}(51$ and $42 \% \mathrm{r}-$ process, respectively) is encouraging; the weaker correlation with Mo may be due to its smaller r-process contribution $(32 \%)$ or due to the smaller number of lines measured (3 vs. 4 and 10) and relatively large abundance errors (Mo has errors due to temperature uncertainties on the order of 0.15 dex, and large continuum errors due to the weak lines). The abundance errors due to the continuum uncertainty, which are as high as 0.3 dex for some stars, may be systematic and could explain the full extent of the deviation from a 1:1 relationship with Pr and Nd. However, based on our error estimates (Table 8) systematic effects could not explain the abundance ranges of $\sim 0.8$ dex we see in the full sample.

Mishenina et al. (2007) also give $[\mathrm{Nd} / \mathrm{Fe}]$ and $[\mathrm{Pr} / \mathrm{Fe}]$ abundances for giant stars. The $[\mathrm{Pr} / \mathrm{Fe}]$ abundances range from +0.4 dex at an $[\mathrm{Fe} / \mathrm{H}]$ of -0.4 to -0.3 dex at an $[\mathrm{Fe} / \mathrm{H}]$ of +0.2 , in good agreement with our stellar $[\mathrm{Pr} / \mathrm{Fe}]$ abundances (the star at $[\mathrm{Fe} / \mathrm{H}]$ of +0.15 and $[\mathrm{Pr} / \mathrm{Fe}]$ of +0.33 is anomalous N6939 star 190, which is also strongly enhanced in $\mathrm{Nd}$ ). However, the $[\mathrm{Nd} / \mathrm{Fe}]$ values for giants fall from +0.3 dex at an $[\mathrm{Fe} / \mathrm{H}]$ of 0.4 to -0.3 dex at an $[\mathrm{Fe} / \mathrm{H}]$ of +0.2 , again showing a 
systematic offset with respect to our data, although the range of the data in this $[\mathrm{Fe} / \mathrm{H}]$ region is about 0.6 dex for both sets of abundances. It is worth noting that we only measured three of the same Nd lines as Mishenina et al. (2007), and the $\log (\mathrm{gf})$ values we used for these lines are systematically lower than theirs by about 0.1 dex. Their measured solar Nd abundance of 1.50 is very similar to ours, but differences in scaling that might not affect solar measurements could still be significant for giants.

Figure 12 shows r-process and mixed element abundances plotted against each other. None of the mixed r- and s-process elements appear to correlate with Eu. Correlations of $\mathrm{Pr}$ and $\mathrm{Nd}$ with $\mathrm{Gd}$ are tighter around the 1:1 line, perhaps due to the subsection of clusters represented in the Gd abundances. Again, it is possible that these correlations could be due to systematic errors, but the fact that the elements with the most similar mix of $\mathrm{r}$ - and s- contributions are the most tightly correlated is suggestive.

\section{SUMMARY AND CONCLUSIONS}

There is still debate over the details of heavy element nucleosynthesis, i.e. the neutron-capture process(es). The site of the r-process has not been conclusively identified, and the yields and relative importance of the known s-process sites are currently being revised due to recent measurements of highly supersolar Ba abundances in young open clusters (e.g., D'Orazi et al. 2009; Maiorca et al. 2012). Old, metal-poor stars and some disk populations have abundance measurements for $\mathrm{r}$ process elements in the literature, but large sets of internally consistent measurements for open clusters are rare. Because r-process elements are thought to be produced in the environments or remnants of massive stars $\left(\mathrm{M}>8 \mathrm{M}_{\odot}\right)$ and s-process elements mainly in low-mass stars $\left(\mathrm{M}<8 \mathrm{M}_{\odot}\right)$, the range and accuracy of determined ages of open clusters make them a valuable tool to study the time scales of production for both sets of elements.

We present here six neutron-capture elements (three primarily r-process, Eu, Gd, and Dy; and three mixed r- and s-process, Mo, Pr, and Nd) for a sample of 68 stars in 23 open clusters. The data are a mixture of spectra previously analyzed by us, obtained from others, and data we have newly gathered. We have used an automated synthesis fitting program of our own devising to reduce the time necessary to measure features with blends and internal structure, and to keep our abundances as homogenous as possible. We are able to measure at least three lines for every element presented, with individual stellar dispersions generally $<0.10 \mathrm{dex}$.

We analyze trends for our sample in $[\mathrm{X} / \mathrm{Fe}]$ vs. cluster age and Galactocentric distance, and find the following:

- Eu, Gd, and Dy have trends with increasing age of $0.024,0.026$, and 0.054 dex $\mathrm{Gyr}^{-1}$, respectively, though the trend in Gd is not statistically significant.

- Mo, Pr, and Nd have age trends consistent with zero, which places them in between positive age trends of pure r-process and negative age trends previously found for Ba.

- All elements except Mo and Gd have significant linear trends with Galactocentric radius, with linear regression slopes $\sim+0.04$ dex $\mathrm{kpc}^{-1}$.

- The relationship of mixed elements (Mo, Pr, Nd) with Galactocentric radius may not be linear, as these appear to be enhanced around $10 \mathrm{kpc}$ and drop around $12 \mathrm{kpc}$. The youngest OCs (age $<2$ Gyr) show no relationship with $\mathrm{R}_{\mathrm{GC}}$, while older clusters show a strong positive correlation for $\mathrm{Pr}$ and Nd.

- For all elements, the scatter at different ages and Galactocentric radii is larger than our cluster dispersions.

The behavior of clusters at large Galactocentric radii (particularly Be 31) presents a puzzle. An enhancement beyond $13 \mathrm{kpc}$ is seen for most elements, though this radius range is sparsely populated in our data. Unfortunately, these clusters are some of the most difficult to observe. Additional clusters at this distance (and additional stars for Be 31) would help distinguish between effects in the different age ranges of clusters (and possible mechanisms for this difference) and overall trends with $\mathrm{R}_{\mathrm{GC}}$. The possible abundance enhancement at 10 kpc may also be an age effect, as it appears to be populated by the youngest clusters, and may even be a sampling effect as the enhancement is not much larger than typical $\mathrm{R}_{\mathrm{GC}}$ dispersions observed in Cepheids. However, since it is seen mainly in the mixed r-and s- elements, pure s-process abundance measurements may elucidate the issue. We will explore s-process Galactic enrichment in an upcoming paper focusing on a set of majority sprocess elements.

J. C. O. gratefully acknowledges support by the Indiana Space Grant Consortium through a Graduate Fellowship. We would like to thank Michael Briley and Laura Magrini for generously allowing us use of McDonald and VLT data, and also APO 3.5m telescope operator Jack Dembicky for help with observing sessions and instrument training. 


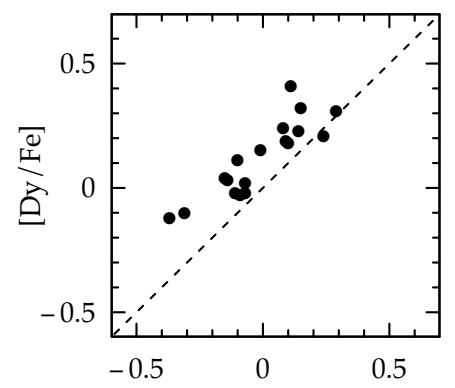

$[\mathrm{Eu} / \mathrm{Fe}]$

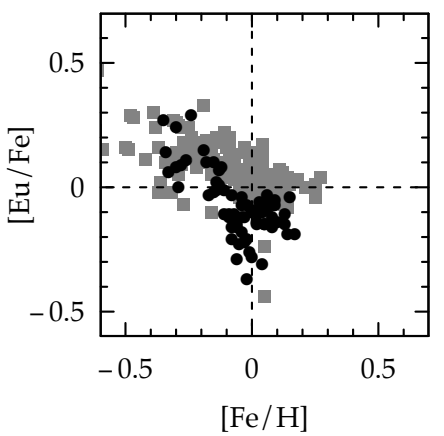

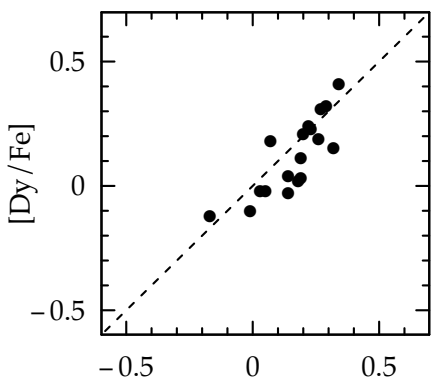

$[\mathrm{Gd} / \mathrm{Fe}]$

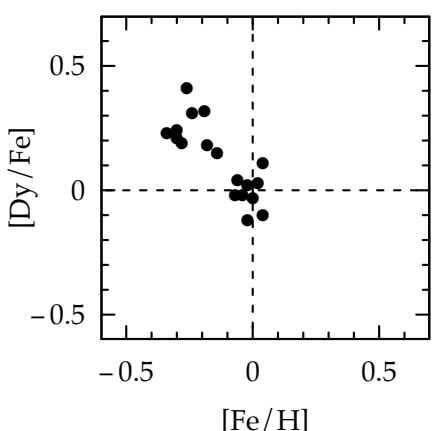

Figure 10. Stellar abundance vs. abundance plots for the r-process elements (Eu, 98\% r; Gd, 82\% r; Dy, 88\% r) and Fe. Circles are our abundances, and squares are disk giant abundances from Mishenina et al. (2007).
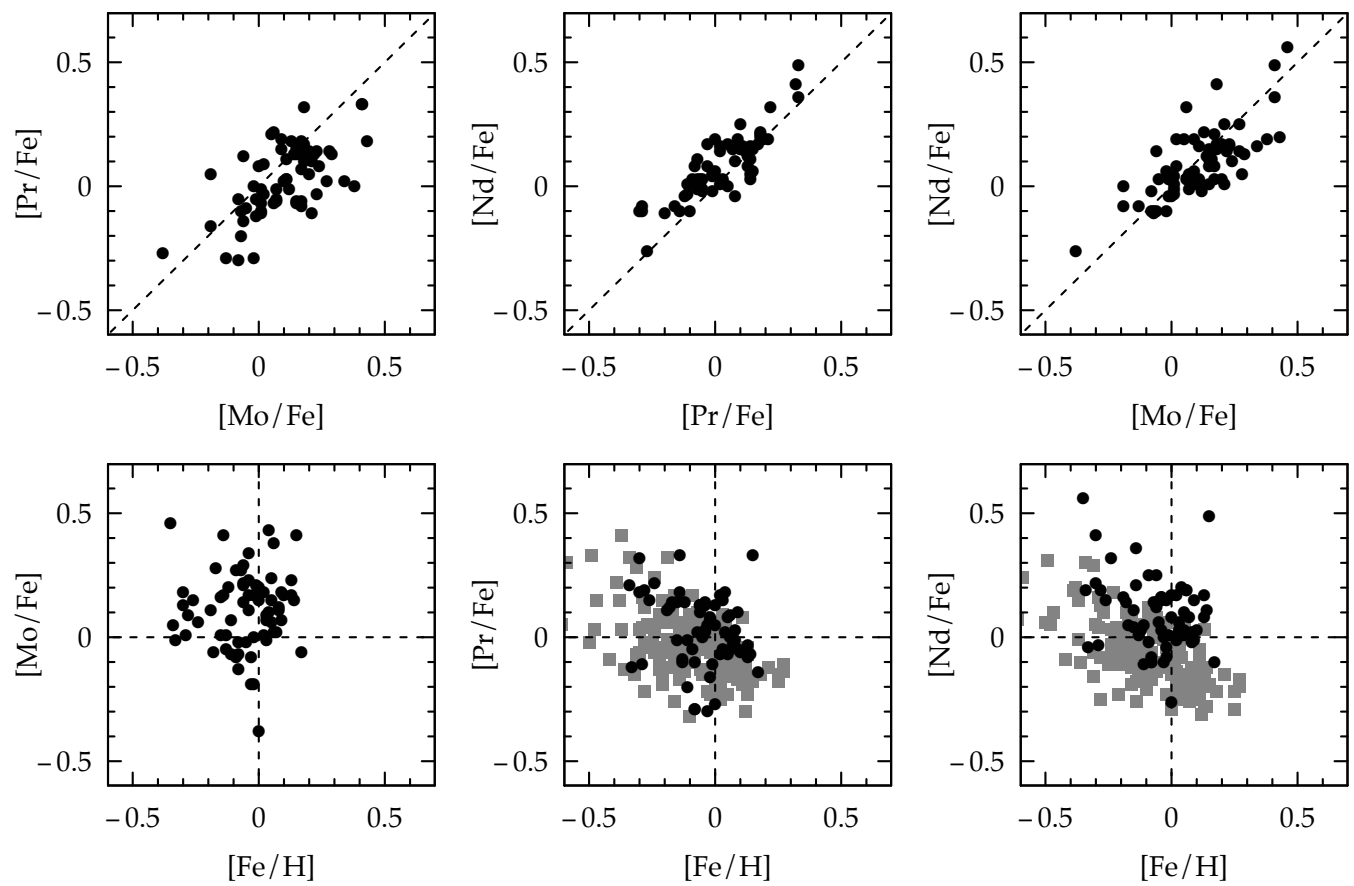

Figure 11. Abundance vs. abundance plots for the mixed elements (Mo, $32 \%$ r; Pr, $51 \%$ r; Nd, $42 \%$ r), and Fe. Symbols are the same as in Fig. 10.

\section{REFERENCES}

Abia, C., Palmerini, S., Busso, M., \& Cristallo, S. 2012, A\&A, 548, A55

Anders, E., \& Grevesse, N. 1989, GeoCoA, 53, 197

Andrievsky, S. M., Bersier, D., Kovtyukh, V. V., et al. 2002a,

A\&A, 384, 140
Andrievsky, S. M., Kovtyukh, V. V., Luck, R. E., et al. 2002b, A\&A, 392, 491

Andrievsky, S. M., Luck, R. E., Martin, P., \& Lépine, J. R. D. 2004, A\&A, 413, 159 

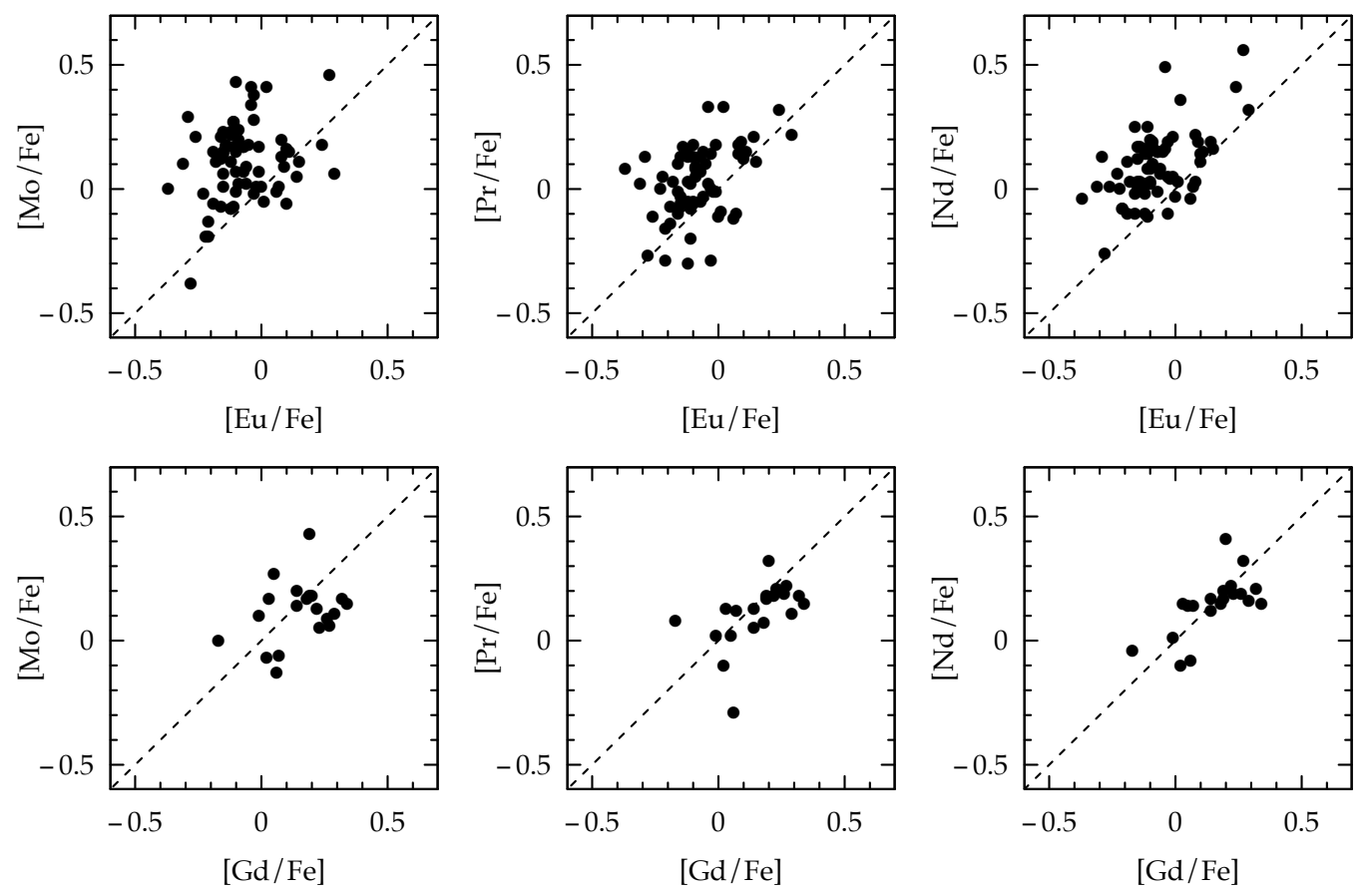

Figure 12. Abundance vs. abundance plots for r-process and mixed elements (Eu, $98 \%$ r; Gd, $82 \%$ r; Mo, $32 \%$ r; Pr, $51 \%$ r; $\mathrm{Nd}, 42 \% \mathrm{r})$.

Andrievsky, S. M., Kovtyukh, V. V., Luck, R. E., et al. 2002c, A\&A, 381, 32

Bird, J. C., Kazantzidis, S., \& Weinberg, D. H. 2012, MNRAS, 420, 913

Bragaglia, A., \& Tosi, M. 2006, AJ, 131, 1544

Burbidge, E. M., Burbidge, G. R., Fowler, W. A., \& Hoyle, F. 1957, Reviews of Modern Physics, 29, 547

Carraro, G., Ng, Y. K., \& Portinari, L. 1998, MNRAS, 296, 1045

Carrera, R., \& Pancino, E. 2011, A\&A, 535, A30

Carretta, E., Bragaglia, A., \& Gratton, R. G. 2007, A\&A, 473, 129

Carretta, E., Bragaglia, A., Gratton, R. G., \& Tosi, M. 2005, A\&A, 441, 131

Cescutti, G., François, P., Matteucci, F., Cayrel, R., \& Spite, M. 2006, A\&A, 448, 557

Cignoni, M., Beccari, G., Bragaglia, A., \& Tosi, M. 2011, MNRAS, 416, 1077

Clariá, J. J., Mermilliod, J.-C., Piatti, A. E., \& Parisi, M. C. 2006, A\&A, 453, 91

De Silva, G. M., Freeman, K. C., Asplund, M., et al. 2007, AJ, 133,1161

Den Hartog, E. A., Lawler, J. E., Sneden, C., \& Cowan, J. J. 2003, ApJS, 148, 543

—. 2006, ApJS, 167, 292

Diehl, R., Halloin, H., Kretschmer, K., et al. 2006, Nature, 439, 45

D’Orazi, V., Magrini, L., Randich, S., et al. 2009, ApJL, 693, L31

François, P., Depagne, E., Hill, V., et al. 2007, A\&A, 476, 935

Freiburghaus, C., Rosswog, S., \& Thielemann, F.-K. 1999, ApJL, 525, L121

Friel, E. D., Jacobson, H. R., Barrett, E., et al. 2003, AJ, 126, 2372

Friel, E. D., Jacobson, H. R., \& Pilachowski, C. A. 2005, AJ, 129,2725

-. 2010, AJ, 139, 1942

Friel, E. D., Janes, K. A., Tavarez, M., et al. 2002, AJ, 124, 2693

Guetter, H. H. 1993, AJ, 106, 220
Gustafsson, B., Edvardsson, B., Eriksson, K., et al. 2008, A\&A, 486,951

Hasegawa, T., Malasan, H. L., Kawakita, H., et al. 2004, PASJ, 56,295

Heiter, U., Lind, K., Asplund, M., et al. 2015, PhyS, 90, 054010

Hinkle, K., Wallace, L., Valenti, J., \& Harmer, D. 2000, Visible and Near Infrared Atlas of the Arcturus Spectrum 3727-9300 A (San Francisco, CA: ASP)

Honda, S., Aoki, W., Kajino, T., et al. 2004, ApJ, 607, 474

Jacobson, H. R., \& Friel, E. D. 2013, AJ, 145, 107

Jacobson, H. R., Friel, E. D., \& Pilachowski, C. A. 2007, AJ, 134,1216

-. 2008, AJ, 135, 2341

-. 2009, AJ, 137, 4753

Jacobson, H. R., Pilachowski, C. A., \& Friel, E. D. 2011, AJ, 142,59

Janes, K. A., \& Phelps, R. L. 1994, AJ, 108, 1773

Kalogera, V., Kim, C., Lorimer, D. R., et al. 2004, ApJL, 601, L179

Lawler, J. E., Wickliffe, M. E., den Hartog, E. A., \& Sneden, C. 2001, ApJ, 563, 1075

Luck, R. E., Gieren, W. P., Andrievsky, S. M., et al. 2003, A\&A, 401, 939

Luck, R. E., \& Lambert, D. L. 2011, AJ, 142, 136

Maciejewski, G., \& Niedzielski, A. 2007, A\&A, 467, 1065

Magrini, L., Randich, S., Zoccali, M., et al. 2010, A\&A, 523, A11

Maiorca, E., Magrini, L., Busso, M., et al. 2012, ApJ, 747, 53

Meggers, W. F., Corliss, C. H., \& Scribner, B. F. 1975, Tables of spectral-line intensities. Part I, II - arranged by elements. (Washington: US GPO)

Mermilliod, J.-C., Latham, D. W., Glushkova, E. V., et al. 2003, A\&A, 399, 105

Milone, A. A. E. 1994, PASP, 106, 1085

Mishenina, T., Pignatari, M., Carraro, G., et al. 2015, MNRAS, 446,3651

Mishenina, T. V., Gorbaneva, T. I., Bienaymé, O., et al. 2007, Astronomy Reports, 51, 382 
Mitschang, A. W., De Silva, G. M., \& Zucker, D. B. 2012, MNRAS, 422, 3527

Mucciarelli, A., Caffau, E., Freytag, B., Ludwig, H.-G., \& Bonifacio, P. 2008, A\&A, 484, 841

Overbeek, J. C., Friel, E. D., Jacobson, H. R., et al. 2015, AJ, 149,15

Pancino, E., Carrera, R., Rossetti, E., \& Gallart, C. 2010, A\&A, 511, A56

Pilachowski, C. A. 1985, PASP, 97, 801

Ramírez, I., \& Allende Prieto, C. 2011, ApJ, 743, 135

Reddy, A. B. S., Giridhar, S., \& Lambert, D. L. 2012, MNRAS, 419,1350

-. 2013, MNRAS, 431, 3338

-. 2015, MNRAS, 450, 4301

Reddy, B. E., Lambert, D. L., \& Allende Prieto, C. 2006, MNRAS, 367, 1329

Reddy, B. E., Tomkin, J., Lambert, D. L., \& Allende Prieto, C. 2003, MNRAS, 340, 304

Salaris, M., Weiss, A., \& Percival, S. M. 2004, A\&A, 414, 163

Schönberner, D., Andrievsky, S. M., \& Drilling, J. S. 2001, A\&A, 366,490

Serminato, A., Gallino, R., Travaglio, C., Bisterzo, S., \& Straniero, O. 2009, PASA, 26, 153

Sneden, C., Cowan, J. J., \& Gallino, R. 2008, ARA\&A, 46, 241

Sneden, C., Lawler, J. E., Cowan, J. J., Ivans, I. I., \& Den Hartog, E. A. 2009, ApJS, 182, 80

Sneden, C., Preston, G. W., McWilliam, A., \& Searle, L. 1994, ApJL, 431, L27
Sneden, C. A. 1973, PhD thesis, THE UNIVERSITY OF TEXAS AT AUSTIN.

Spite, M., \& Spite, F. 1978, A\&A, 67, 23

Tautvaišienè, G., Edvardsson, B., Puzeras, E., Barisevičius, G., \& Ilyin, I. 2010, MNRAS, 409, 1213

Tautvaišienè, G., Edvardsson, B., Puzeras, E., \& Ilyin, I. 2005, A\&A, 431, 933

Tautvaišienè, G., Edvardsson, B., Tuominen, I., \& Ilyin, I. 2000, A\&A, 360, 499

Travaglio, C., Gallino, R., Arnone, E., et al. 2004, ApJ, 601, 864

Twarog, B. A., Ashman, K. M., \& Anthony-Twarog, B. J. 1997, AJ, 114, 2556

Vande Putte, D., Garnier, T. P., Ferreras, I., Mignani, R. P., \& Cropper, M. 2010, MNRAS, 407, 2109

Vangioni, E., Goriely, S., Daigne, F., François, P., \& Belczynski, K. 2016, MNRAS, 455, 17

Wasserburg, G. J., Busso, M., \& Gallino, R. 1996, ApJL, 466, L109

Wehmeyer, B., Pignatari, M., \& Thielemann, F.-K. 2015, MNRAS, 452, 1970

Whaling, W., \& Brault, J. W. 1988, PhyS, 38, 707

Wickliffe, M. E., Lawler, J. E., \& Nave, G. 2000, JQSRT, 66, 363

Wu, Z.-Y., Zhou, X., Ma, J., \& Du, C.-H. 2009, MNRAS, 399, 2146

Yong, D., Carney, B. W., \& Friel, E. D. 2012, AJ, 144, 95

Yong, D., Carney, B. W., \& Teixera de Almeida, M. L. 2005, AJ, 130,597 